\title{
REFLEXIVE HULL DISCRIMINANTS AND APPLICATIONS
}

\author{
KENNETH CHAN, JASON GADDIS, ROBERT WON, AND JAMES J. ZHANG
}

\begin{abstract}
We introduce the reflexive hull discriminant as a tool to study noncommutative algebras that are finitely generated, but not necessarily free, over their centers. As an example, we compute the reflexive hull discriminants for quantum generalized Weyl algebras and use them to determine automorphism groups and other properties, recovering results of Suárez-Alvarez, Vivas, and others.
\end{abstract}

\section{INTRODUCTION}

The discriminant is a powerful tool in the study of noncommutative algebras. It has proved useful in computing automorphism groups and solving isomorphism problems [14, 15, 16, 18, 22], resolving the Zariski cancellation problem for different families of noncommutative algebras [6, 21, 35], and studying the representations of Azumaya algebras [13, 53, 52]. Nguyen, Trampel, and Yakimov have also established a correspondence between discriminants and Poisson geometry [38].

In general, the discriminant can be difficult to compute by hand, although formulas exist for special cases [13, 18, 17, 20, 38, 39. To apply these results one typically needs the algebra to be a finite rank free module over its center (although this is not necessary in [18]). Otherwise, one can use the notion of modified discriminant ideals, introduced in [15. In this case, the discriminant can be defined to be the gcd of the elements in the modified discriminant ideal. This more general notion shares many properties with the earlier definition, and they coincide for an algebra that is a free module over its center. However, the gcd of the elements in the modified discriminant ideal may not exist. To overcome this deficiency, we introduce the reflexive hull discriminant.

Throughout let $\mathbb{k}$ be a base field. Suppose $A$ is a finitely generated (=affine) prime $\mathbb{k}$-algebra that is a finitely generated module over its center $Z$ (also called module-finite over $Z$ for short) and let $\operatorname{MD}(A / Z) \subseteq Z$ denote the modified discriminant ideal of $A / Z$ [Definition [1.2(3)]. We say $A / Z$ satisfies the principal closure condition (or $P C C$ for short) if there exists a normal element $d \in A$ such that $\operatorname{MD}(A / Z) A \subseteq d A$ and $\operatorname{GKdim}(d A / \mathrm{MD}(A / Z) A) \leq \operatorname{GKdim} A-2$ (where GKdim denotes Gelfand-Kirillov dimension). Let $(-)^{\vee}$ denote the $Z$-dual functor $\operatorname{Hom}_{Z}(-, Z)$. If $Z$ is a Cohen-Macaulay (CM) domain and $A / Z$ satisfies the PCC (plus some mild hypotheses), then Lemma 1.5 shows that $(\operatorname{MD}(A / Z) A)^{\vee \vee}=d A$. In this case, $d$ is called an extended reflexive hull discriminant (or $\overline{\mathcal{R}}$-discriminant for short) of $A$ over $Z$, and is denoted $\bar{\varrho}(A / Z)$. We also have a "non-extended" version of a reflexive hull discriminant (or $\mathcal{R}$-discriminant for short) of $A$ over $Z$ which is denoted by $\varrho(A / Z)$ (see Section 1 for more details).

The $\overline{\mathcal{R}}$-discriminant (or $\mathcal{R}$-discriminant) has the advantage of not requiring the existence of gcds (e.g., of the elements in the modified discriminant ideal). Even in the situation that a gcd of the elements in the modified discriminant ideal exists, the $\overline{\mathcal{R}}$-discriminant is often easier to compute because it can be computed locally. However, in general, it is not clear when an $\overline{\mathcal{R}}$-discriminant

2020 Mathematics Subject Classification. 16E65, 16P99, 16W99, 16W20,11R29.

Key words and phrases. Modified discriminant, reflexive hull discriminant, quantized generalized Weyl algebra, automorphism problem, cancellation problem. 
exists, even for connected graded noetherian Calabi-Yau algebras which are module-finite over their centers [Question 4.9]. We are able to show that $\overline{\mathcal{R}}$-discriminants exist for certain skew polynomial rings over their centers.

Definition 0.1. Fix an integer $n \geq 2$. Let $\mathbf{p} \in M_{n}\left(\mathbb{k}^{\times}\right)$be a multiplicatively antisymmetric matrix (so $p_{i i}=1$ and $p_{i j}=p_{j i}^{-1}$ for all $1 \leq i<j \leq n$ ). The skew polynomial ring $\mathbb{k}_{\mathbf{p}}\left[x_{1}, \ldots, x_{n}\right]$ is the $\mathbb{k}$-algebra generated by $\left\{x_{1}, \ldots, x_{n}\right\}$ and subject to relations

$$
x_{j} x_{i}=p_{i j} x_{i} x_{j}, \quad 1 \leq i<j \leq n .
$$

The algebra $\mathbb{k}_{\mathbf{p}}\left[x_{1}, \ldots, x_{n}\right]$ is an Artin-Schelter regular algebra of global dimension $n$

Theorem 0.2 (Theorem 4.6). Let $A$ be a skew polynomial ring $\mathbb{k}_{\mathbf{p}}\left[x_{1}, \ldots, x_{n}\right]$ where each $p_{i j}$ is a root of unity. Then an $\overline{\mathcal{R}}$-discriminant of $A$ over its center exists.

Note that the $\overline{\mathcal{R}}$-discriminant shares several nice properties with the (modified) discriminant. In many applications, if the (modified) discriminant ideal is not principal, one can use the $\overline{\mathcal{R}}$ discriminant instead. We denote the set of the units of a ring $A$ by $A^{\times}$and for $f, g \in A$, we write $f={ }_{A^{\times}} g$ if $f=c g$ for some $c \in A^{\times}$. The following result shows that, like the earlier notions of the discriminant, the $\overline{\mathcal{R}}$-discriminant can be used to study automorphisms and locally nilpotent derivations.

Theorem 0.3 (Theorem 1.11). Assume that $A$ is a prime algebra with center $Z$. Assume further that $Z$ is an affine $C M$ domain and that Hypothesis 1.1 holds for $(A, Z)$. Suppose $d$ is an $\mathcal{R}$ discriminant of $A$ over $Z$.

(1) If $g \in \operatorname{Aut}(A)$, then $g(d)={ }_{A^{\times}} d$.

(2) Suppose that char $\mathbb{k}=0$ and that $A^{\times}=\mathbb{k}^{\times}$. If $\delta$ is a locally nilpotent derivation of $A$, then $\delta(d)=0$.

The following result is useful in computing the $\overline{\mathcal{R}}$-discriminant of a tensor product. A slightly modified version is proved in Theorem 2.6 in geometric language.

Theorem 0.4 (Theorem 1.14). Let $A, A^{\prime}$ be prime algebras which are module-finite over their centers $Z, Z^{\prime}$ respectively. Let $w=\operatorname{rk}(A / Z)$ and $w^{\prime}=\operatorname{rk}\left(A^{\prime} / Z^{\prime}\right)$. Assume that $A \otimes A^{\prime}$ is prime and that Hypotheses 1.1(2) and 1.10 hold for $(A, Z)$ and $\left(A^{\prime}, Z^{\prime}\right)$. Suppose $d$ (resp. $\left.d^{\prime}\right)$ is an $\overline{\mathcal{R}}$-discriminant of $A / Z$ (resp. $\left.A^{\prime} / Z^{\prime}\right)$. Then $d^{w^{\prime}} \otimes\left(d^{\prime}\right)^{w}$ is an $\overline{\mathcal{R}}$-discriminant of $\left(A \otimes A^{\prime}\right) /\left(Z \otimes Z^{\prime}\right)$.

The notion of the $\overline{\mathcal{R}}$-discriminant which we introduce here is a very natural generalization of the classical discriminant. In [18], the authors introduced a $p$-power discriminant to handle the automorphism and cancellation problems for Veronese subrings of skew polynomial rings. Similarly we briefly introduce the $p$-power reflexive hull discriminant (respectively, extended $p$-power reflexive hull discriminant), denoted $\mathcal{R}_{v}^{p}$-discriminant (respectively, $\overline{\mathcal{R}}_{v}^{p}$-discriminant), see Section 3 , Several other generalizations of the classical discriminant exist in the literature, which we illustrate in the following diagram. 


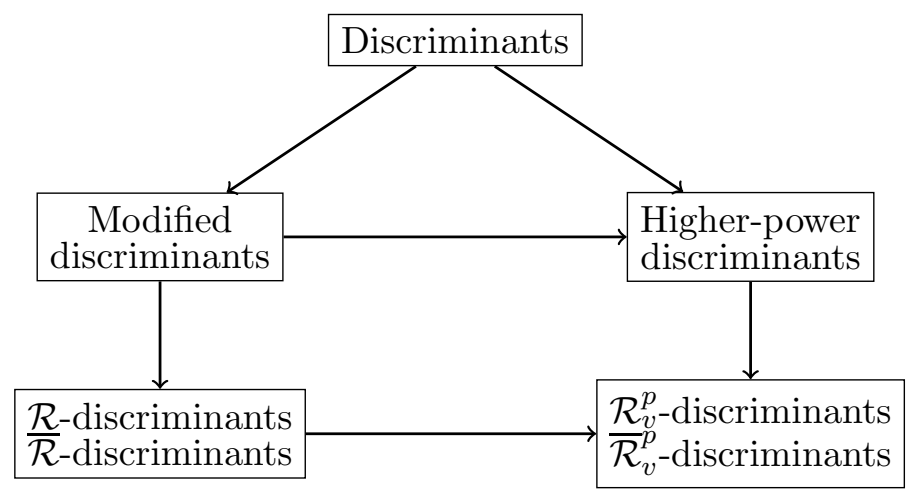

To illustrate the power of the notion of the $\overline{\mathcal{R}}$-discriminant, we study the $\overline{\mathcal{R}}$-discriminant for a certain family of generalized Weyl algebras (GWAs) [Definition 0.5. The GWA construction is sufficiently general as to encompass many families of well-known algebras, including the classical Weyl algebras and primitive quotients of $U\left(\mathfrak{s l}_{2}\right)$ [2]. Much of the theory of GWAs so far generalizes well-known properties of those families of algebras, see [25, 26, 27, 28, 42, 45, 47]. The subclass known as quantum GWAs includes quantum planes and quantum Weyl algebras. A fundamental problem for any class of algebras is to study their automorphisms and isomorphisms within that class. These questions have been addressed for quantum GWAs (of degree one) in a number of papers [4, 41, 50] with the assumption that the base ring has Krull dimension one. In this paper we compute the automorphism groups for certain higher degree quantum GWAs and recover a number of earlier results in the degree one case.

Definition 0.5. Let $R$ be a ring, $\sigma \in \operatorname{Aut}(R)$, and $h$ a nonzero central element of $R$. The generalized Weyl algebra ( $G W A$ ) of degree one $R(x, y, \sigma, h)$ is generated over $R$ by $x$ and $y$ modulo the relations

$$
x y=h, \quad y x=\sigma^{-1}(h), \quad x r=\sigma(r) x, \quad y r=\sigma^{-1}(r) y,
$$

for all $r \in R$. We say the GWA $R(x, y, \sigma, h)$ is quantum if $R=\mathbb{k}[t]$ and $\sigma(t)=q t$ for some $q \in \mathbb{k}^{\times}$.

In Section 5.2, we compute the reflexive hull discriminant of a degree one quantum GWA $W$ with parameter a root of unity (which is not 1 ). In this case, when $\operatorname{deg}_{t}(h)>1$, the center $Z$ of $W$ is a hypersurface singularity and $W$ is not free over $Z$. In general, both $W$ and $Z$ may have infinite global dimension.

Theorem 0.6 (Theorem 5.11). Let $W:=\mathbb{k}[t](x, y, \sigma, h)$ be a quantum $G W A$ of degree one with

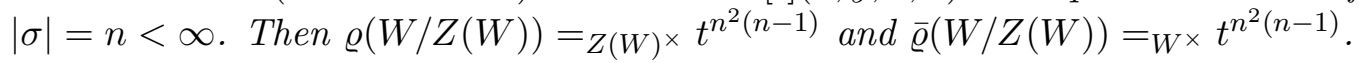

Theorem 0.6 generalizes several known results. When $h=t$, then $W$ is a quantum plane and when $h=t-1$, then $W$ is isomorphic to a quantum Weyl algebra. Our result recovers the discriminant in both of these special cases [14, 17].

We also compute the $\mathcal{R}-(\overline{\mathcal{R}}-)$ discriminant for a class of higher degree GWAs [Theorem 5.14 . We mention one application here.

Definition 0.7. An algebra $A$ is cancellative if for any algebra $B, A[x] \cong B[x]$ implies that $A \cong B$.

Theorem 0.8. Let $W:=\mathbb{k}[t](\mathbf{x}, \mathbf{y}, \sigma, h)$ be a quantum $G W A$ of degree $m>1$ (see Definition 5.1).

(1) (Theorem 5.14) Suppose that each $q_{i}$ is a root of unity with $n_{i}=\left|\sigma_{i}\right|<\infty, \operatorname{gcd}\left(n_{i}, n_{j}\right)=1$ for all $i \neq j$, and $n_{i}>1$ for at least one $i$. Then the $\mathcal{R}$-discriminant of $W$ over its center exists and $\varrho(W / Z(W))={ }_{Z(W)} \times t^{n^{2}(n-1)}$, where $n^{2}$ is the rank of $W$ over its center. 
(2) (Theorem[6.6(2)]) Every tensor product of finitely many GWAs as in part (1) and Theorem 0.6 is cancellative.

Remark 0.9. In this paper we mainly consider algebras that are module-finite over their centers. For algebras that are not module-finite over their centers, one could either

(a) reduce the situation to the module-finite case by using mod $p$ reduction, or

(b) use other versions of the discriminant (e.g., the $\mathcal{P}$-discriminants introduced in [35]) and their reflexive hulls.

This paper is organized as follows. We introduce the notion of an $\mathcal{R}-(\overline{\mathcal{R}}$ - $)$ discriminant in Section 1 and give a geometric interpretation in Section [2. In Section 3 we briefly introduce the notion of an $\mathcal{R}_{v^{-}}^{p}\left(\overline{\mathcal{R}}_{v^{-}}^{p}\right)$ discriminant for positive integers $p$ and $v$. Theorem 0.2 is proved in Section 4 . In Section 5 we prove Theorem 0.6 and in Subsection 5.3 we prove a version of Theorem 0.6. namely, Theorem 0.8(1), for GWAs of higher degree. We conclude, in Section 6, by demonstrating several applications of $\mathcal{R}-(\overline{\mathcal{R}}-)$ discriminants. We compute the automorphism group of a quantum GWA, recovering a result of Suárez-Alvarez and Vivas [50]. We also recover their result that the set of locally nilpotent derivations of a quantum GWA is trivial. We further extend these results to tensor products of quantum GWAs and higher degree quantum GWAs.

Acknowledgments. R. Won was partially supported by an AMS-Simons Travel Grant and J. J. Zhang was partially supported by the US National Science Foundation (Nos. DMS-1700825 and DMS-2001015).

\section{Discriminants, MOdified Discriminants, And $\mathcal{R}-(\overline{\mathcal{R}}-)$ DisCRiminants}

The goal of this section is to recall the definition of the (modified) discriminant and introduce a new notion, called the (extended) reflexive hull discriminant. Let $\mathbb{k}$ be a base field. All algebras will be assumed to be $\mathbb{k}$-algebras unless otherwise stated, and we write $\otimes$ for $\otimes_{\mathbb{k}}$.

Let $R$ be a commutative domain, let $B$ be an $R$-algebra, and assume that $R$ is a subalgebra of $B$. When $R$ is the center of $B$, we often use $Z$ instead of $R$. Let $F$ be a localization of $R$ such that $B_{F}:=B \otimes_{R} F$ is finitely generated and free over $F$ with $w=\operatorname{rk}_{F}\left(B_{F}\right)<\infty$. There is a natural embedding of $R$-algebras given by left-multiplication:

$$
\operatorname{lm}: B \rightarrow B_{F} \rightarrow \operatorname{End}_{F}\left(B_{F}\right) \cong M_{w}(F)
$$

Let $\operatorname{tr}_{\text {int }}$ denote the usual (internal) matrix trace in $M_{w}(F)$. The regular trace is the composition

$$
\operatorname{tr}_{\text {reg }}: B \stackrel{\operatorname{lm}}{\longrightarrow} M_{w}(F) \stackrel{\operatorname{tr}_{\text {int }}}{\longrightarrow} F .
$$

In this paper, $\operatorname{tr}$ denotes the regular trace, unless otherwise stated. We remark that this is merely for convenience, and one could also use the standard trace or reduced trace map, see [13, Section $2.2]$, instead. We often use the following hypothesis.

Hypothesis 1.1. Let $(B, R)$ satisfy the following conditions.

(1) $B$ is a prime $\mathbb{k}$-algebra containing $R$ as a central subalgebra such that $B$ is a finitely generated $R$-module.

(2) The image of tr is contained in $R$. 
Hypothesis 1.1(2) is essential for several results in this paper. By [40, Theorem 10.1], if $B$ is a prime ring and $R$ is the center of $B$ such that $R$ is a normal domain, then $(B, R)$ satisfies Hypothesis 1.1(2). In the applications given in this paper, Hypothesis 1.1(2) can be checked easily. A general comment can be found in Lemma 4.2(2).

Suppose $B$ is a prime ring. A regular normal element $x \in B$ divides $y \in B$ if $y=b x$ for some $b \in B$. If $\mathcal{S}$ is a subset of $B$, then an element $x \in B$ is a common divisor of $\mathcal{S}$ if (i) $x$ is a regular normal element and (ii) $x$ divides every $z \in \mathcal{S}$. Furthermore, $x$ is the greatest common divisor $($ gcd) of $\mathcal{S}$ if any common divisor $y$ of $\mathcal{S}$ divides $x$. The $\operatorname{gcd}$ of $\mathcal{S}$, if it exists, is unique up to a unit in $B$.

We now recall several definitions introduced in [15].

Definition 1.2. [15, Definition 1.2] Assume $(B, R)$ satisfies Hypothesis 1.1, For a positive integer $v$, let $\mathcal{U}=\left\{u_{i}\right\}_{i=1}^{v}$ and $\mathcal{U}^{\prime}=\left\{u_{i}^{\prime}\right\}_{i=1}^{v}$ be $v$-element subsets of $B$.

(1) The discriminant of the pair $\left(\mathcal{U}, \mathcal{U}^{\prime}\right)$ is defined to be

$$
d_{v}\left(\mathcal{U}, \mathcal{U}^{\prime}\right)=\operatorname{det}\left(\operatorname{tr}\left(u_{i} u_{j}^{\prime}\right)_{i, j=1}^{v}\right) \in R .
$$

(2) The $v$-discriminant ideal $D_{v}(B / R)$ is the ideal in $R$ generated by the set of elements $d_{v}(\mathcal{U}, \mathcal{U})$ where $\mathcal{U}$ ranges over all $v$-element subsets of $B$.

(3) The modified $v$-discriminant ideal $\mathrm{MD}_{v}(B / R)$ is the ideal in $R$ generated by the set of elements $d_{v}\left(\mathcal{U}, \mathcal{U}^{\prime}\right)$ where $\mathcal{U}, \mathcal{U}^{\prime}$ range over all $v$-element subsets of $B$. If $B$ is a finitely generated $R$-module of rank $w$ then we use the notation $\operatorname{MD}(B / R):=\operatorname{MD}_{w}(B / R)$.

(4) The $v$-discriminant $d_{v}(B / R)$ is the gcd in $B$, if it exists, of the elements in $\operatorname{MD}_{v}(B / R)$.

(5) The extended $v$-discriminant ideal $\overline{\mathrm{MD}}_{v}(B / R)$ is the ideal in $B$ generated by $\operatorname{MD}_{v}(B / R)$, namely, $\overline{\mathrm{MD}}_{v}(B / R)=\mathrm{MD}_{v}(B / R) B$. Similarly, we use the notation $\overline{\mathrm{MD}}(B / R)$ to denote the ideal $\mathrm{MD}(B / R) B$.

In the special case that $B$ is free over $R$ of rank $w$, we have $D_{w}(B / R)=\operatorname{MD}_{w}(B / R)$ generated by a single element $d(B / R):=d_{w}(B / R)$, which we call the discriminant of $B$ over $R$.

Here, we introduce a new variant of the discriminant which will be useful for algebras that are finitely generated, but not necessarily free, over their centers.

Let $M$ be a module over a fixed commutative domain $R$. Define $M^{\vee}=\operatorname{Hom}_{R}(M, R)$. The reflexive hull of $M$ is defined to be $M^{\vee \vee}$. There is a natural $R$-morphism $\iota: M \rightarrow M^{\vee \vee}$ defined by

$$
\iota(x)(f)=f(x)
$$

for all $x \in M, f \in M^{\vee}$. It is well-known that if $I$ is an ideal of $R$, then $I^{\vee \vee}$ is an ideal of $R$. In fact, if $B$ is a prime algebra that is a finitely generated reflexive module over a central noetherian subalgebra and if $M$ is an ideal of $B$, then $M^{\vee \vee}$ is an ideal of $B$ containing $M$.

Definition 1.3. Retain the above notation. Let $(B, R)$ satisfy Hypothesis 1.1,

(1) The $\mathcal{R}$-discriminant ideal (or reflexive hull discriminant ideal) of $B$ over $R$ is defined to be

$$
\mathcal{R}(B / R):=(\mathrm{MD}(B / R))^{\vee \vee} \subseteq R .
$$

(2) If, further, $\mathcal{R}(B / R)$ is a principal ideal of $R$ generated by an element $d$, then $d$ is called an $\mathcal{R}$-discriminant (or reflexive hull discriminant) of $B$ over $R$ and denoted by $\varrho(B / R)$.

(3) Suppose $B$ is a reflexive $R$-module. The $\overline{\mathcal{R}}$-discriminant ideal (or extended reflexive hull discriminant ideal) of $B$ over $R$ is defined to be

$$
\overline{\mathcal{R}}(B / R):=(\overline{\mathrm{MD}}(B / R))^{\vee \vee} \subseteq B .
$$


(4) If, further, $\overline{\mathcal{R}}(B / R)$ is a principal ideal of $B$ generated by a normal element $d$, then $d$ is called an $\overline{\mathcal{R}}$-discriminant (or extended reflexive hull discriminant) of $B$ over $R$ and denoted by $\bar{\varrho}(B / R)$.

It is clear that $\varrho(B / R)$ (resp. $\varrho(B / R)$ ), if it exists, is unique up to a unit in $R$ (resp. $B$ ).

If $R$ is a UFD (e.g., any localization of the commutative polynomial ring), then every reflexive ideal of $R$ is principal and so $\varrho(B / R)$ always exists. Similarly, if $B$ is a noncommutative noetherian UFR, then $\bar{\varrho}(B / R)$ exists under mild conditions (see Theorem 4.8).

Next, we introduce some conditions that are closely related to the existence of the $\mathcal{R}$-discriminant, as well as weak $\mathcal{R}$ - $(\overline{\mathcal{R}}$-)discriminants. These notions can be computationally useful. Throughout, we use Gelfand-Kirillov dimension, denoted by GKdim, as our dimension function. We refer the reader to [30, 37] for definitions and basic properties related to Gelfand-Kirillov dimension. Our use of GK dimension is not essential, and other exact dimension functions work equally well.

Definition 1.4. Let $(B, R)$ satisfy Hypothesis 1.1. Let $A$ be an algebra (which may be $B, R$, or another algebra).

(1) We say an ideal $I \subseteq A$ satisfies the principal closure condition (or $P C C$ ) if there exists a normal element $d \in A$ such that

(a) $I \subseteq d A=A d$, and

(b) $\operatorname{GKdim}(d A / I) \leq \operatorname{GKdim} A-2$.

(2) We say $B / R$ satisfies the reflexive discriminant condition (or $R D C$ for short) if $\mathrm{MD}(B / R) \subseteq$ $R$ satisfies PCC for some nonzero element $d \in R$. In this case $d$ is called a weak $\mathcal{R}$ discriminant of $B$ over $R$.

(3) We say $B / R$ satisfies the extended reflexive discriminant condition (or ERDC for short) if $\overline{\mathrm{MD}}(B / R) \subseteq B$ satisfies PCC for a normal element $d \in B$. In this case $d$ is called a weak $\overline{\mathcal{R}}$-discriminant of $B$ over $R$.

The element $d$ in either part (1) or (2) or (3) (if it exists) may not be unique (even up to a unit) in general, unless $R$ is CM [Lemma 1.5].

In many examples, RDC and ERDC are practical conditions to use. We will show that under some mild conditions, RDC (ERDC) implies the existence of a $\mathcal{R}-(\overline{\mathcal{R}}-)$ discriminant.

Recall from [12, Definitions 4.1 and 4.2] that the (homological) grade of a nonzero $R$-module $M$ is defined to be

$$
j(M)=\min \left\{i \mid \operatorname{Ext}_{R}^{i}(M, R) \neq 0\right\} .
$$

We say that $R$ is $G K$-Macaulay if, for all nonzero finitely generated $R$-modules $M$, we have $\operatorname{GKdim}(M)+j(M)=\operatorname{GKdim}(R)$. If $R$ is an affine commutative domain, then $R$ being GKMacaulay is equivalent to $R$ being CM, see [12, Theorem 4.8(i) $\Leftrightarrow($ iv)].

Lemma 1.5. Let $(B, R)$ satisfy Hypothesis 1.1. Suppose that $R$ is an affine $C M$ domain and that $B$ is a $C M$ reflexive module over $R$.

(1) Let $M$ be an ideal of $B$ satisfying PCC with respect to $d \in B$. Then $M^{\vee \vee}$ is a principal ideal of $B$ generated by $d$.

(2) Let $I$ be an ideal of $R$ satisfying $P C C$ with respect to $d \in R$. Then $I^{\vee \vee}$ is a principal ideal of $R$ generated by $d$.

(3) Suppose $B / R$ satisfies $R D C$ with respect to $d \in R$. Then

$$
\operatorname{MD}(B / R)^{\vee \vee}=d R \quad \text { and } \quad \varrho(B / R)=R_{6} \times d .
$$


(4) Suppose $B / R$ satisfies $E R D C$ with respect to $d \in B$. Then

$$
(\overline{\mathrm{MD}}(B / R))^{\vee \vee}=d B \quad \text { and } \quad \bar{\varrho}(B / R)={ }_{B^{\times}} d .
$$

Proof. (1) By definition, there is a short exact sequence

$$
0 \rightarrow M \rightarrow d B \rightarrow d B / M \rightarrow 0 .
$$

Applying $(-)^{\vee}=\operatorname{Hom}_{R}(-, R)$ gives

$$
0 \rightarrow \operatorname{Hom}_{R}(d B / M, R) \rightarrow \operatorname{Hom}_{R}(d B, R) \rightarrow \operatorname{Hom}_{R}(M, R) \rightarrow \operatorname{Ext}_{R}^{1}(d B / M, R) .
$$

Since $R$ is CM (and hence GK-Macaulay by [12, p.1451] or [12, Theorem $4.8(\mathrm{i}) \Leftrightarrow(\mathrm{iv})$ ]) and $M$ satisfies $\mathrm{PCC}$ with respect to $d$, we have $\operatorname{GKdim}(d B / M) \leq \operatorname{GKdim} B-2=\operatorname{GKdim} R-2$. Therefore, $j(d B / M) \geq 2$ by the GK-Macaulay property. By definition, $\operatorname{Hom}_{R}(d B / M, R)=\operatorname{Ext}_{R}^{1}(d B / M, R)=$ 0 . Hence

$$
M^{\vee}=\operatorname{Hom}_{R}(M, R)=\operatorname{Hom}_{R}(d B, R)=(d B)^{\vee}=d^{-1} B^{\vee} .
$$

Applying $(-)^{\vee}$ again yields the desired isomorphism.

(2) This is a special case of part (1) by taking $B=R$.

(3) This follows from part (2).

(4) This follows from part (1).

In all applications given in this paper, the hypotheses in Lemma 1.5 will be verified.

A useful property of the various flavors of discriminants is their invariance under automorphisms (see [14, Lemma 1.8], [15, Lemma 1.4]). We next show that the $\mathcal{R}$-( $\overline{\mathcal{R}}$-)discriminant is preserved, up to a unit, by any automorphism.

Lemma 1.6. Suppose $B$ is an algebra with center $R$ and suppose that $(B, R)$ satisfy Hypothesis 1.1. Assume additionally that $R$ is a domain. Let $G$ be a group of algebra automorphisms of $B$.

(1) If $I$ is a $G$-invariant ideal of $R$, then $I^{\vee \vee}$ is also a $G$-invariant ideal of $R$.

(2) Suppose $B$ is reflexive over $R$. Let $I$ be a G-invariant ideal of $B$. Then $I^{\vee \vee}$ is also a $G$-invariant ideal of $B$.

Proof. It suffices to prove (2), since (1) follows from (2) by setting $B=R$. The induced action of $G$ on $I^{\vee}=\operatorname{Hom}_{R}(I, R)$ is given as follows: for any $g \in G$ and $\varphi \in I^{\vee}$, we have $(g \varphi)(x)=g\left(\varphi\left(g^{-1}(x)\right)\right)$. Similarly $I^{\vee \vee}$ has an induced $G$-action.

Since $B$ is reflexive, the map $\iota: B \rightarrow B^{\vee \vee}$ as in (E1.2.1) is an isomorphism. Then $I^{\vee \vee}$ is the subset of elements $x \in B$ such that $\iota(x)\left(I^{\vee}\right) \subseteq R$. To show that $I^{\vee \vee}$ is $G$-invariant, it suffices to show that the induced action of $G$ on $I^{\vee \vee}$ is the same as the $G$-action inherited as a subset of $R$. For $x \in I$ and $\varphi \in I^{\vee}$, we have

$$
(g \iota(x))(\varphi)=g\left(\iota(x)\left(g^{-1} \varphi\right)\right)=g\left(\left(g^{-1} \varphi\right)(x)\right)=g g^{-1}(\varphi(g(x)))=\varphi(g(x))=\iota(g(x))(\varphi),
$$

which proves the claim.

The $\mathcal{R}$ - $(\overline{\mathcal{R}}$ - $)$ discriminant utilizes reflexive hulls to obtain a good principal ideal in the case that the modified discriminant ideal $\mathrm{MD}(A / Z)$ is not principal. It may be possible that other operations can be employed in similar ways.

Question 1.7. What are some other examples of closure operations on ideals (integral closure, Frobenius closure, tight closure, etc) which send $G$-invariant ideals to $G$-invariant ideals? 
In the remainder of this section, we are interested in the modified discriminant ideals and $\mathcal{R}$ discriminants for tensor products of algebras. In order to study these $\mathcal{R}$-discriminants, we recall the definition of a quasi-basis from [15. We refine the definition slightly here by including the data of a generating set (the set $X$ below) as part of the data of a quasi-basis.

Definition 1.8. [15, Definition 1.10] Let $B$ be an algebra which is module-finite over a central subalgebra $R$. Let $F$ be the field of fractions of $R$ (or a localization of $R$ ). Suppose that $B_{F}:=$ $B \otimes_{R} F$ is a finite dimensional $F$-vector space. A set $\mathbf{b}=\left\{b_{1}, \ldots, b_{w}\right\} \subseteq B$ is a quasi-basis of $B$ with respect to a finite set $X=\left\{x_{j}\right\}_{j \in J} \subseteq B$ if the following four conditions hold:

(a) $\mathbf{b} \subseteq X$,

(b) $\mathbf{b}$ is an $F$-basis of $B_{F}$, where $b_{i}$ is viewed as $b_{i} \otimes 1 \in B_{F}$,

(c) $X$ generates $B$ as an $R$-module, and

(d) each $x_{j}$ is in the union of one-dimensional $F$-subspaces $\bigcup_{i=1}^{w} F b_{i}$.

By part (d), for each $j \in J$, we can write $x_{j}=c_{j} b_{i}$ for some $i$ (uniquely determined by $j$ ) and some scalar $c_{j} \in F$. Let $C$ be the set of all such nonzero coefficients $c_{j}$. If only conditions (a)-(c) hold, we call $\mathbf{b}$ a semi-basis of $B$ with respect to $X$.

By Definition 1.8(d), we may define a map $f: J \rightarrow\{1, \ldots, w\}$ where $f(j)$ is defined to be the $i$ such that $x_{j} \in F b_{i}$. We say $I \subseteq J$ is a $w$-restricted subset if $|I|=w$ and $f(I)=\{1, \ldots, w\}$.

Lemma 1.9. [15, Lemma 1.11(1)] Let $B$ be an algebra which is module-finite over a central subalgebra $R$. Let $X$ be a generating set of $B$ over $R$ and let $\mathbf{b}$ be a quasi-basis of $B$ with respect to $X$. Then, as ideals in $R$,

$$
\operatorname{MD}(B / R)=d_{w}(\mathbf{b}, \mathbf{b})\left\langle c_{I} c_{K} \mid I, K\right\rangle .
$$

where $I, K$ range over $w$-restricted subsets of $J$ and where $c_{I}=\prod_{j \in I} c_{j}$ and $c_{K}=\prod_{j \in K} c_{j}$.

Although many of the results above hold for more general $(B, R)$, for much of the remainder of the paper, we will consider the specific case when $R$ is the center of $B$. Henceforth, we will often use the notation $(A, Z)$ where $A$ is an algebra with center $Z$.

Hypothesis 1.10. Let $A$ be an algebra with center $Z$. Suppose that $(A, Z)$ satisfy Hypothesis 1.1 and further suppose that:

(1) $Z$ is affine and CM, and

(2) there is a quasi-basis with respect to a finite generating set of the $Z$-module $A$.

A derivation of an algebra $A$ is a $\mathbb{k}$-linear map $\delta: A \rightarrow A$ satisfying the Leibniz rule:

$$
\delta(a b)=\delta(a) b+a \delta(b)
$$

for all $a, b \in A$. A derivation $\delta$ is called locally nilpotent if for every $a \in A$ there exists $n \in \mathbb{N}$ such that $\delta^{n}(a)=0$.

Theorem 1.11. Suppose $(A, Z)$ satisfies Hypothesis 1.10(1).

(1) Suppose $d$ is an $\mathcal{R}$-discriminant of $A$ over $Z$. If $g \in \operatorname{Aut}(A)$, then $g(d)={ }_{Z \times} d$.

(2) Suppose $d$ is an $\overline{\mathcal{R}}$-discriminant of $A$ over $Z$. If $g \in \operatorname{Aut}(A)$, then $g(d)=A_{A^{\times}} d$.

(3) Let $d$ be either $\varrho(A / Z)$ or $\bar{\varrho}(A / Z)$. Suppose that char $\mathbb{k}=0$ and that $A^{\times}=\mathbb{k}^{\times}$. If $\delta$ is a locally nilpotent derivation of $A$, then $\delta(d)=0$. 
Proof. (1) Let $g \in \operatorname{Aut}(A)$. Then $g$ naturally preserves the center $Z$ of $A$. By [15, Lemma 1.4], $g$ preserves the modified discriminant ideal $\operatorname{MD}(A / Z)$. Since $\operatorname{MD}(A / Z)^{\vee} \vee=d Z$, then the result follows from Lemma 1.6 .

(2) The proof is similar to the proof of part (1).

(3) This result is completely analogous to [15, Proposition 1.5].

We will use the following notation for tensor products of finite sets. If $\mathbf{b} \subseteq A$ and $\mathbf{b}^{\prime} \subseteq A^{\prime}$ are finite sets, then we define $\mathbf{b} \otimes \mathbf{b}^{\prime}=\left\{a \otimes b \mid a \in \mathbf{b}, b \in \mathbf{b}^{\prime}\right\} \subseteq A \otimes A^{\prime}$.

An algebra is called $P I$ if it satisfies a polynomial identity.

Lemma 1.12. Let $A$ and $A^{\prime}$ be prime $P I$ algebras with centers $Z$ and $Z^{\prime}$, respectively, such that Hypothesis 1.1 holds for both $(A, Z)$ and $\left(A^{\prime}, Z^{\prime}\right)$. Let $w=\operatorname{rk}(A / Z)$ and $w^{\prime}=\operatorname{rk}\left(A^{\prime} / Z^{\prime}\right)$. Assume that $A \otimes A^{\prime}$ is prime (so that the center is a domain). Suppose $\mathbf{b}=\left\{b_{i}\right\}_{i=1}^{w}$ and $\mathbf{b}^{\prime}=\left\{b_{i}^{\prime}\right\}_{i=1}^{w^{\prime}}$ are quasi-bases for $A$ and $A^{\prime}$ with respect to the (finite) generating sets $\mathbf{x}=\left\{x_{j}\right\}_{j \in J}$ and $\mathbf{x}^{\prime}=\left\{x_{j}^{\prime}\right\}_{j \in J^{\prime}}$, respectively. Then the following hold.

(1) The set $\mathbf{b} \otimes \mathbf{b}^{\prime}$ is a quasi-basis of $A \otimes A^{\prime}$ with respect to $\mathbf{x} \otimes \mathbf{x}^{\prime}$.

(2) $\mathrm{MD}(A / Z)^{w^{\prime}} \otimes \mathrm{MD}\left(A^{\prime} / Z^{\prime}\right)^{w}=\mathrm{MD}\left(\left(A \otimes A^{\prime}\right) /\left(Z \otimes Z^{\prime}\right)\right)$. As a consequence,

$$
\overline{\mathrm{MD}}(A / Z)^{w^{\prime}} \otimes \overline{\mathrm{MD}}\left(A^{\prime} / Z^{\prime}\right)^{w}=\overline{\mathrm{MD}}\left(\left(A \otimes A^{\prime}\right) /\left(Z \otimes Z^{\prime}\right)\right) .
$$

(3) If $\operatorname{MD}(A / Z) \subseteq d Z$ and $\operatorname{MD}\left(A^{\prime} / Z^{\prime}\right) \subseteq d^{\prime} Z^{\prime}$ for some elements $d \in Z$ and $d^{\prime} \in Z^{\prime}$, respectively, then

$$
\operatorname{MD}\left(\left(A \otimes A^{\prime}\right) /\left(Z \otimes Z^{\prime}\right)\right) \subseteq\left(d^{w^{\prime}} \otimes\left(d^{\prime}\right)^{w}\right)\left(Z \otimes Z^{\prime}\right) .
$$

(4) If $\overline{\mathrm{MD}}(A / Z) \subseteq d Z$ and $\overline{\mathrm{MD}}\left(A^{\prime} / Z^{\prime}\right) \subseteq d^{\prime} Z^{\prime}$ for some normal elements $d \in A$ and $d^{\prime} \in A^{\prime}$, respectively, then

$$
\overline{\mathrm{MD}}\left(\left(A \otimes A^{\prime}\right) /\left(Z \otimes Z^{\prime}\right)\right) \subseteq\left(d^{w^{\prime}} \otimes\left(d^{\prime}\right)^{w}\right)\left(A \otimes A^{\prime}\right) .
$$

Proof. It is easy to see that $Z\left(A \otimes A^{\prime}\right)=Z \otimes Z^{\prime}$. Part (1) follows directly from the definition of quasi-basis, and parts $(3,4)$ are consequences of part $(2)$. It remains to show part $(2)$.

To prove $(2)$, we use the generators of the ideal $\mathrm{MD}(B / R)$ given by Lemma 1.9 to identify $\operatorname{MD}\left(\left(A \otimes A^{\prime}\right) /\left(Z \otimes Z^{\prime}\right)\right)$ with the tensor product $\operatorname{MD}(A / Z)^{w^{\prime}} \otimes \operatorname{MD}\left(A^{\prime} / Z^{\prime}\right)^{w}$.

As above, we define a map $f: J \rightarrow\{1, \ldots, w\}$ by letting $f(\alpha)$ be the unique index such that $x_{\alpha}=c_{\alpha} b_{f(\alpha)}$. We define $f^{\prime}: J^{\prime} \rightarrow\left\{1, \ldots, w^{\prime}\right\}$ similarly so that $x_{\alpha}^{\prime}=c_{\alpha}^{\prime} b_{f^{\prime}(\alpha)}^{\prime}$. Then $f \times f^{\prime}: J \times J^{\prime} \rightarrow$ $\{1, \ldots, w\} \times\left\{1, \ldots, w^{\prime}\right\}$ is well-defined. Adopting the notation that if $I \subseteq J$ then $c_{I}=\prod_{i \in I} c_{I}$ (and similarly for subsets of $J^{\prime}$ and $J \times J^{\prime}$ ), we can write out the generators of the modified discriminant ideals explicitly:

$$
\begin{aligned}
\operatorname{MD}(A / Z) & \left.=d_{w}(\mathbf{b}, \mathbf{b})\left\langle c_{I} c_{K}\right| w \text {-restricted subsets } I, K \subseteq J\right\rangle \\
\operatorname{MD}\left(A^{\prime} / Z^{\prime}\right)= & \left.d_{w^{\prime}}\left(\mathbf{b}^{\prime}, \mathbf{b}^{\prime}\right)\left\langle c_{I^{\prime}}^{\prime} c_{K^{\prime}}^{\prime}\right| w^{\prime} \text {-restricted subsets } I^{\prime}, K^{\prime} \subseteq J^{\prime}\right\rangle \\
\operatorname{MD}\left(\left(A \otimes A^{\prime}\right) /\left(Z \otimes Z^{\prime}\right)\right)= & d_{w w^{\prime}}\left(\mathbf{b} \otimes \mathbf{b}^{\prime}, \mathbf{b} \otimes \mathbf{b}^{\prime}\right) \\
& \left.\left\langle\gamma_{M} \gamma_{N}\right| w w^{\prime} \text {-restricted subsets } M, N \subseteq J \times J^{\prime}\right\rangle
\end{aligned}
$$

where $\gamma_{M}=\prod_{(\alpha, \beta) \in M} c_{\alpha} \otimes c_{\beta}^{\prime}$ for $M \subseteq J \times J^{\prime}$. The following equality

$$
d_{w w^{\prime}}\left(\mathbf{b} \otimes \mathbf{b}^{\prime}, \mathbf{b} \otimes \mathbf{b}^{\prime}\right)=d_{w}(\mathbf{b}, \mathbf{b})^{w^{\prime}} \otimes d_{w^{\prime}}\left(\mathbf{b}^{\prime}, \mathbf{b}^{\prime}\right)^{w}
$$

is elementary, as it follows from properties of Kronecker products of matrices, so we omit its justification. 
Let $\mathrm{pr}_{1}$ and $\mathrm{pr}_{2}$ denote the projections of $J \times J^{\prime}$ to $J$ and $J^{\prime}$, respectively. Suppose that $M, N$ are $w w^{\prime}$-restricted subsets $J \times J^{\prime}$. For each $n \in\{1,2, \ldots, w\}$ and $n^{\prime} \in\left\{1,2, \ldots, w^{\prime}\right\}$ we define

$$
\begin{aligned}
I_{n}^{\prime} & =\left(f \circ \mathrm{pr}_{1}\right)^{-1}(n) \cap M & K_{n}^{\prime} & =\left(f \circ \mathrm{pr}_{1}\right)^{-1}(n) \cap N \\
I_{n^{\prime}} & =\left(f^{\prime} \circ \mathrm{pr}_{2}\right)^{-1}\left(n^{\prime}\right) \cap M & K_{n^{\prime}} & =\left(f^{\prime} \circ \mathrm{pr}_{2}\right)^{-1}\left(n^{\prime}\right) \cap N .
\end{aligned}
$$

Note that since $M$ and $N$ are $w w^{\prime}$-restricted subsets of $J \times J^{\prime}$, we have, for each $n, n^{\prime}$, that $\left|I_{n^{\prime}}\right|=\left|K_{n^{\prime}}\right|=w$ and $\left|I_{n}^{\prime}\right|=\left|K_{n}^{\prime}\right|=w^{\prime}$ (and these are $w$ and $w^{\prime}$-restricted subsets of $J$ and $J^{\prime}$, respectively). Then

$$
\gamma_{M} \gamma_{N}=\prod_{n^{\prime}=1}^{w^{\prime}} c_{I_{n^{\prime}}} c_{K_{n^{\prime}}} \otimes \prod_{n=1}^{w} c_{I_{n}^{\prime}}^{\prime} c_{K_{n}^{\prime}}^{\prime} .
$$

Hence, $d_{w w^{\prime}}\left(\mathbf{b} \otimes \mathbf{b}^{\prime}, \mathbf{b} \otimes \mathbf{b}^{\prime}\right) \gamma_{M} \gamma_{N} \in \operatorname{MD}(A / Z)^{w^{\prime}} \otimes \operatorname{MD}\left(A^{\prime} / Z^{\prime}\right)^{w}$. Conversely, let $C$ denote the right hand side of (E1.12.1) where $I_{n^{\prime}}, K_{n^{\prime}}$ are $w$-restricted subsets of $J$ and $I_{n}^{\prime}, K_{n}^{\prime}$ are $w^{\prime}$-restricted subsets of $J^{\prime}$. Then we can express $C$ in the form $C=\gamma_{\widetilde{M}} \gamma_{\widetilde{N}}$ where

$$
\begin{aligned}
& \widetilde{M}=\bigcup_{n^{\prime}=1}^{w^{\prime}} \bigcup_{n=1}^{w}\left(I_{n^{\prime}} \times J^{\prime}\right) \cap\left(J \times I_{n}^{\prime}\right) \\
& \widetilde{N}=\bigcup_{n^{\prime}=1}^{w^{\prime}} \bigcup_{n=1}^{w}\left(K_{n^{\prime}} \times J^{\prime}\right) \cap\left(J \times K_{n}^{\prime}\right) .
\end{aligned}
$$

Indeed, for each $1 \leq n \leq w$ and $1 \leq n^{\prime} \leq w^{\prime}$, we can write $\left(I_{n^{\prime}} \times J^{\prime}\right) \cap\left(J \times I_{n}^{\prime}\right)$ (somewhat perversely) as the singleton $\left(\left(\left.f\right|_{I_{n^{\prime}}}\right)^{-1}(n),\left(\left.f^{\prime}\right|_{I_{n}^{\prime}}\right)^{-1}\left(n^{\prime}\right)\right)$ since $I_{n^{\prime}}$ being a $w$-restricted subset of $J$ means $\left.f\right|_{I_{n^{\prime}}}$ (and similarly $\left.f\right|_{I_{n}^{\prime}}$ ) is invertible. This shows that $\widetilde{M}$ and $\widetilde{N}$ are $w w^{\prime}$-restricted subsets of $J \times J^{\prime}$. It follows from the definition that $\left(f \circ \operatorname{pr}_{1}\right)^{-1}(n) \cap \widetilde{M}=I_{n}^{\prime}$ and $\left(f^{\prime} \circ \operatorname{pr}_{2}\right)^{-1}\left(n^{\prime}\right) \cap \widetilde{M}=I_{n^{\prime}}($ similar statements hold for $\tilde{N}, K_{n}^{\prime}$ and $\left.K_{n^{\prime}}\right)$.

This verifies the formula $C=\gamma_{\widetilde{M}} \gamma_{\widetilde{N}}$ which shows that

$$
d_{w}(\mathbf{b}, \mathbf{b})^{w^{\prime}} d_{w^{\prime}}\left(\mathbf{b}, \mathbf{b}^{\prime}\right)^{w} C \in \operatorname{MD}\left(\left(A \otimes A^{\prime}\right) /\left(Z \otimes Z^{\prime}\right)\right) .
$$

This completes the proof of the first equation of (2). The second equation is an immediate consequence of the first.

Lemma 1.13. Let $A$ be a noetherian prime PI algebra. Suppose $M_{1}, M_{2}$ are commuting ideals of $A$ and $d_{1}, d_{2}$ are commuting normal elements of $A$ such that, for $i=1,2$,

(a) $M_{i} \subseteq d_{i} A$ and

(b) $\operatorname{GKdim}\left(d_{i} A / M_{i}\right) \leq \operatorname{GKdim}(A)-2$.

Then

(1) $M_{1} M_{2} \subseteq d_{1} d_{2} A$ and

(2) $\operatorname{GKdim}\left(d_{1} d_{2} A / M_{1} M_{2}\right) \leq \operatorname{GKdim}(A)-2$.

Proof. Clearly we can assume that $d_{1} d_{2} \neq 0$. By replacing $M_{i}$ by $d_{i}^{-1} M_{i}$ we can assume that $d_{i}=1$ (for $i=1,2$ ). We get a short exact sequence

$$
0 \rightarrow M_{1} / M_{1} M_{2} \rightarrow A / M_{1} M_{2} \rightarrow A / M_{1} \rightarrow 0 .
$$


By hypothesis (b), $\operatorname{GKdim}\left(A / M_{1}\right) \leq \operatorname{GKdim}(A)-2$ and since $M_{1} / M_{1} M_{2}$ is a finitely generated $A / M_{1}$-module, we also have

$$
\operatorname{GKdim}\left(M_{1} / M_{1} M_{2}\right) \leq \operatorname{GKdim}\left(A / M_{2}\right) \leq \operatorname{GKdim}(A)-2 .
$$

Hence by additivity of GKdim on short exact sequences, we conclude that $\operatorname{GKdim}\left(A / M_{1} M_{2}\right) \leq$ $\operatorname{GKdim}(A)-2$.

Theorem 1.14. Retain the hypothesis of Lemma 1.12.

(1) Suppose that $A / Z$ (resp. $A^{\prime} / Z^{\prime}$ ) satisfies $R D C$ with respect to $d$ (resp. $d^{\prime}$ ), a weak $\mathcal{R}$ discriminant of $A / Z$ (resp. $\left.A^{\prime} / Z^{\prime}\right)$. Then $\left(A \otimes A^{\prime}\right) /\left(Z \otimes Z^{\prime}\right)$ satisfies $R D C$ with respect to $d^{w^{\prime}} \otimes\left(d^{\prime}\right)^{w}$, which is a weak $\mathcal{R}$-discriminant of $\left(A \otimes A^{\prime}\right) /\left(Z \otimes Z^{\prime}\right)$. If further Hypothesis 1.10(1) holds for $\left(A \otimes A^{\prime}, Z \otimes Z^{\prime}\right)$, then $d^{w^{\prime}} \otimes\left(d^{\prime}\right)^{w}=\left(Z \otimes Z^{\prime}\right) \times \varrho\left(\left(A \otimes A^{\prime}\right) /\left(Z \otimes Z^{\prime}\right)\right)$.

(2) Suppose that $A / Z$ (resp. $A^{\prime} / Z^{\prime}$ ) satisfies ERDC with respect to $d$ (resp. $d^{\prime}$ ), a weak $\overline{\mathcal{R}}$ discriminant of $A / Z$ (resp. $\left.A^{\prime} / Z^{\prime}\right)$. Then $\left(A \otimes A^{\prime}\right) /\left(Z \otimes Z^{\prime}\right)$ satisfies ERDC with respect to $d^{w^{\prime}} \otimes\left(d^{\prime}\right)^{w}$, which is a weak $\overline{\mathcal{R}}$-discriminant of $\left(A \otimes A^{\prime}\right) /\left(Z \otimes Z^{\prime}\right)$. If further Hypothesis 1.10 (1) holds for $\left(A \otimes A^{\prime}, Z \otimes Z^{\prime}\right)$, then $d^{w^{\prime}} \otimes\left(d^{\prime}\right)^{w}=_{\left(A \otimes A^{\prime}\right) \times} \bar{\varrho}\left(\left(A \otimes A^{\prime}\right) /\left(Z \otimes Z^{\prime}\right)\right)$.

Proof. (1) Let $R=Z \otimes Z^{\prime}, M_{1}=\operatorname{MD}(A / Z)^{w^{\prime}} \otimes Z^{\prime}$ and $M_{2}=Z \otimes \operatorname{MD}\left(A^{\prime} / Z^{\prime}\right)^{w}$. Let $d_{1}=d^{w^{\prime}}$ and $d_{2}=\left(d^{\prime}\right)^{w}$. The first assertion follows from Lemmas 1.12 and 1.13 applied to $R$.

Since $Z$ and $Z^{\prime}$ are affine noetherian, then so is $R$. Moreover, $Z$ and $Z^{\prime}$ are CM so $R$ is as well by [8, Theorem 2.1]. The second assertion now follows from Lemma 1.5(1) and the first assertion.

(2) The proof of this assertion is analogous to the proof of part (1).

In [20], the authors studied discriminants of twisted tensor products of algebras.

Question 1.15. Under similar hypotheses to those in [20], is it possible to compute the $\mathcal{R}-(\overline{\mathcal{R}}-)$ discriminant of a twisted tensor product of two algebras?

To conclude this section, we make an observation. It is easy to see that if $d$ is a weak $\mathcal{R}$ discriminant of $B$ over $R$ [Definition 1.4(2)], then it is a weak $\overline{\mathcal{R}}$-discriminant of $B$ over $R$ [Definition [.4(2)]. Note that if $R$ is an affine CM normal domain, then the $\mathcal{R}$-discriminant (resp. $\overline{\mathcal{R}}$-discriminant) exists if and only if the weak $\mathcal{R}$-discriminant (resp. $\overline{\mathcal{R}}$-discriminant) exists [Lemmas 1.5 and 2.4. Combining the above two sentences, under some mild hypothesis, if $\varrho(B / R)$ exists, then so does $\bar{\varrho}(B / R)$ and $\bar{\varrho}(B / R)=\varrho(B / R)$.

Lemma 1.16. Assume Hypothesis 1.1 for $(A, Z)$. Suppose that $Z$ is an affine $C M$ normal domain and that $A$ is reflexive over $Z$. If $\varrho(A / Z)$ exists, then so does $\bar{\varrho}(A / Z)$ and $\bar{\varrho}(A / Z)={ }_{A^{\times}} \varrho(A / Z)$.

\section{A GeOMetric interpretation}

In this section we provide a geometric motivation for the reflexive hull discriminant. For convenience, we assume the next hypothesis for a large part of this section.

Hypothesis 2.1. Let $(A, Z)$ satisfy Hypothesis 1.1, Let $X:=\operatorname{Spec} Z$ be an affine integral normal $\mathbb{k}$-variety. Let $\mathcal{D}$ (resp. $\overline{\mathcal{D}}$ ) be the sheafification of the ideal $\mathrm{MD}(A / Z)$ in $Z$ (resp. $\overline{\mathrm{MD}}(A / Z)$ in $A$ ).

We are interested in the reflexive hull of the modified discriminant ideal $\mathcal{D}$ of the $\mathcal{O}_{X}$-order $A$ and consider it as a subsheaf of $\mathcal{O}_{X}$. Note that $\mathcal{D}^{\vee \vee}$ is, by definition, a reflexive sheaf of rank one, so we can construct it using the following well-known fact [46, Lemma 0AY6]. 
Lemma 2.2. Let $X$ be an integral locally noetherian normal scheme. Let $\mathcal{L}$ be a coherent $\mathcal{O}_{X^{-}}$ module. The following are equivalent:

(a) $\mathcal{L}$ is reflexive,

(b) there exists an open subscheme $\iota: U \rightarrow X$ such that

(b1) every irreducible component of $X \backslash U$ has codimension $\geq 2$ in $X$,

(b2) $\iota^{*} \mathcal{L}$ is finite locally free, and

(b3) $\mathcal{L}=\iota_{*} \iota^{*} \mathcal{L}$.

Let $\mathcal{L}_{1}$ and $\mathcal{L}_{2}$ be two coherent reflexive $\mathcal{O}_{X}$-modules. By Lemma 2.2, $\mathcal{L}_{1} \cong \mathcal{L}_{2}$ if there is an open subscheme $\iota: U \subseteq X$ such that $X \backslash U$ has codimension $\geq 2, \iota^{*} \mathcal{L}_{1}$ and $\iota^{*} \mathcal{L}_{2}$ are locally free, and $\iota^{*} \mathcal{L}_{1} \cong \iota^{*} \mathcal{L}_{2}$. The following lemma is a special case of Lemma 2.2, and is useful in computing reflexive hull discriminants.

Lemma 2.3. Suppose $(A, Z)$ satisfies Hypothesis [2.1. Let $U$ be an open subset of $X$ such that $X \backslash U$ has codimension $\geq 2$.

(1) If there exists a normal element $d \in A$ such that the principal ideal ( $d$ ) of $A$ agrees with $\overline{\mathrm{MD}}(A / Z)$ on $U$, then $\bar{\varrho}(A / Z)={ }_{A^{\times}} d$.

(2) Similarly, if there exists an element $d \in Z$ such that the principal ideal ( $d)$ of $Z$ agrees with $\operatorname{MD}(A / Z)$ on $U$, then $\varrho(A / Z)={ }_{Z^{\times}} d$.

The following lemma is easy.

Lemma 2.4. Let $A$ be an $\mathcal{O}_{X}$-order and assume $(A, Z)$ satisfies Hypothesis 2.1.

(1) Suppose that $A$ is a $C M Z$-module. Let $\mathfrak{m} \in X$ be a regular closed point. Then

$$
\operatorname{MD}\left(A_{\mathfrak{m}} / Z_{\mathfrak{m}}\right)=\operatorname{MD}(A / Z)_{\mathfrak{m}}=\left(\operatorname{MD}(A / Z)_{\mathfrak{m}}\right)^{\vee \vee}
$$

is a principal, hence reflexive, ideal of $Z_{\mathfrak{m}}$. As a consequence, the support of $\mathcal{D}^{\vee \vee} / \mathcal{D}$ has codimension $\geq 2$, or equivalently, $\operatorname{GKdim}\left(\operatorname{MD}(A / Z)^{\vee \vee} / \mathrm{MD}(A / Z)\right) \leq \operatorname{GKdim} Z-2$.

(2) Suppose that $A$ is a $C M$ normal $Z$-module. Let $\mathfrak{m} \in X$ be a regular closed point. Then

$$
\overline{\operatorname{MD}}\left(A_{\mathfrak{m}} / Z_{\mathfrak{m}}\right)=\overline{\operatorname{MD}}(A / Z)_{\mathfrak{m}}=\left(\overline{\mathrm{MD}}(A / Z)_{\mathfrak{m}}\right)^{\vee \vee}
$$

is a principal, hence reflexive, ideal of $A_{\mathfrak{m}}$. As a consequence, the support of $\overline{\mathcal{D}}^{\vee \vee} / \overline{\mathcal{D}}$ has codimension $\geq 2$, or equivalently, $\operatorname{GKdim}\left(\overline{\mathrm{MD}}(A / Z)^{\vee \vee} / \overline{\mathrm{MD}}(A / Z)\right) \leq \operatorname{GKdim} A-2$.

(3) Suppose that $V$ is an affine open subset of $X$ such that $\left.A\right|_{V}$ is locally free. Then

$$
\left.\operatorname{MD}(A / Z)\right|_{V}=\operatorname{MD}\left(\left.A\right|_{V} / \mathcal{O}(V)\right)=\mathcal{O}_{V}(D)
$$

where $D$ is given by the zero locus of the usual discriminant $d \in \mathcal{O}_{V}(V)$.

Proof. (1) Since $\mathfrak{m}$ is a smooth closed point, $Z_{\mathfrak{m}}$ is local and regular. Since $A$ is CM over $Z, A_{\mathfrak{m}}$ is $\mathrm{CM}$ over $Z_{\mathfrak{m}}$. By the Auslander-Buchsbaum formula, $A_{\mathfrak{m}}$ is a finite projective (hence free) module over $Z_{\mathfrak{m}}$. So the (modified) discriminant ideal is principal and hence reflexive.

The consequence is clear.

(2) The proof is similar to the proof of part (1).

(3) By commutative algebra, taking reflexive hulls commutes with localization as does the formation of modified discriminants. Thus the assertion follows. 
Let $U$ be the maximal open subvariety such that $\left.\mathcal{D}\right|_{U}$ is locally free. When $A$ is CM over $Z$, then $U$ contains the non-singular locus of $X$ by Lemma 2.4(1) (so the $\mathcal{R}$-discriminant exists over an open subvariety whose complement has codimension $\geq 2$ ). We can write $\left.\mathcal{D}\right|_{U}=\left.\mathcal{D}^{\vee \vee}\right|_{U}=\mathcal{O}_{U}(D)$ where $D$ is a Cartier divisor on $U$. Denote by $\iota: U \rightarrow X$ the inclusion map, then $\mathcal{D}^{\vee \vee}=\iota_{*} \mathcal{O}_{U}(D)$ by Lemma 2.2. Therefore one might consider this Cartier divisor as a "shadow" of the reflexive hull discriminant $\mathcal{D}^{\vee \vee}$. In other words, the $\mathcal{R}$-discriminant is a "completion" or "closure" of this Cartier divisor. A similar comment can be made for $\overline{\mathcal{D}}$ which is considered as a subsheaf of (the sheafification of) $A$. It is interesting to work out the closed subvarieties so that $\mathcal{D}$ (resp. $\mathcal{D}^{\vee \vee}, \overline{\mathcal{D}}$, and $\overline{\mathcal{D}}^{\vee \vee}$ ) are not locally free.

The upshot of Lemmas 2.3 and 2.4 is that to compute $\mathcal{D}^{\vee \vee}$ (resp. $\overline{\mathcal{D}}^{\vee \vee}$ ) we do not have to compute the modified discriminant ideal, i.e., we do not have to consider all $\operatorname{rk}(A / Z) \times \operatorname{rk}(A / Z)$ minors of a large matrix. This reduces the computation significantly. Once we have determined $D$ as a Cartier divisor on $U$ (or equivalently, a locally free ideal of $\mathcal{O}(U)$ ), the question of whether the reflexive hull of the modified discriminant ideal is principal becomes a question in algebraic geometry: is $\iota_{*} \mathcal{O}_{U}(D)$ locally free? Or equivalently, does the Cartier divisor $D$ on $U$ extend to a Cartier divisor on all of $X$ ? We illustrate this recipe by computing the $\mathcal{R}$-discriminant of several classes of algebras in the following examples as well as in Sections 4 and 5 ,

Example 2.5. Suppose char $\mathbb{k} \neq 2$. Let $A=\mathbb{k}_{\mathbf{p}}\left[x_{1}, x_{2}, x_{3}\right]$ as in Definition 0.1 (for $n=3$ ). Note that $\varrho(A / Z)$ exists by Theorem 4.6 .

(1) Let $\left(p_{12}, p_{13}, p_{23}\right)=(-1,1,1)$. The center of $A$ is $Z=\mathbb{k}[u, v, w]$ where $u=x_{1}^{2}, v=x_{2}^{2}$, and $w=x_{3}$. The rank of $A$ over $Z$ is 4 . The discriminant, modified discriminant, and reflexive hull discriminant are all equal to $\varrho(A / Z)=\bar{\varrho}(A / Z)=_{\mathbb{k}^{\times}}(u v)^{2}=x_{1}^{4} x_{2}^{4}$.

(2) Let $\left(p_{12}, p_{13}, p_{23}\right)=(-1,-1,1)$. The center of $A$ is $\mathbb{k}[u, v, w, z] /\left(v w-z^{2}\right)$ where $u=x_{1}^{2}$, $v=x_{2}^{2}, w=x_{3}^{2}$, and $z=x_{2} x_{3}$. The rank of $A$ over $Z$ is 4 . Let $V$ be the open subset of $X$ with $v \neq 0$. Over $V$, the discriminant of $A_{v}$ is $(u v)^{2}$, or simply $u^{2}$, since $v^{2}$ is a unit in $Z_{v}$. Let $W$ be the open subset of $X$ with $w \neq 0$. Similarly, over $W$, the discriminant of $A_{w}$ is $u^{2}$. Note that $X \backslash(V \cup W)$ has codimension 2 , so the $\mathcal{R}$-discriminant is $\varrho(A / Z)=\bar{\varrho}(A / Z)==_{\mathbb{k}^{\times}} u^{2}=x_{1}^{4}$, which agrees with the modified discriminant given in [15, Example 1.3(3)]. The modified discriminant ideal is generated by $\left\{x_{1}^{4} x_{2}^{i} x_{3}^{4-i}\right\}_{i=0}^{4}$ by [15, Example 1.3(3)].

(3) Let $\left(p_{12}, p_{13}, p_{23}\right)=(-1,-1,-1)$. The center of $A$ is $\mathbb{k}[u, v, w, z] /\left(u v w-z^{2}\right)$ where $u=x_{1}^{2}$, $v=x_{2}^{2}, w=x_{3}^{2}$, and $z=x_{1} x_{2} x_{3}$. Let $U_{3}$ be the open subset of $X$ with $u v \neq 0$. Over $U_{3}$, the discriminant of $A_{u v}$ is $\left(x_{1} x_{2}\right)^{4}$ which is equivalent to 1 . Let $U_{2}$ (resp. $U_{1}$ ) be the open subset of $X$ with $u w \neq 0$ (resp, $v w \neq 0$ ). Since $X \backslash\left(U_{1} \cup U_{2} \cup U_{3}\right)$ has codimension 2 in $X$, the $\mathcal{R}$-discriminant of $A / Z$ is $\varrho(A / Z)=\bar{\varrho}(A / Z)=_{\mathbb{R} \times 1}$.

To conclude this section we prove a geometric version of Theorem 1.14,

Theorem 2.6. Let $\mathbb{k}$ be an algebraically closed field. Suppose that

(a) A is a prime algebra that is module-finite over its center $Z$,

(b) $Z$ is an affine normal $C M$ domain and that $\varrho(A / Z)$ exists, and

(c) $A$ is $C M$ as a module over $Z$.

Let $\left(A^{\prime}, Z^{\prime}\right)$ be another pair satisfying (a)-(c). Let $w\left(\right.$ resp. $\left.w^{\prime}\right)$ be the rank of $A$ (resp. $\left.A^{\prime}\right)$ over $Z$ (resp. $Z^{\prime}$ ). Further assume that $A \otimes A^{\prime}$ is prime. Then the following hold.

(1) $Z \otimes Z^{\prime}$ is a normal $C M$ domain.

(2) $A \otimes A^{\prime}$ is a prime ring with center $Z \otimes Z^{\prime}$ and $A \otimes A^{\prime}$ is $C M$ as a module over $Z \otimes Z^{\prime}$. 


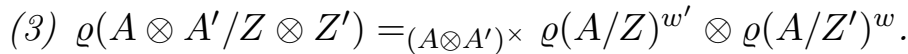

(3') Suppose $A \otimes A^{\prime}$ is reflexive over $Z \otimes Z^{\prime}$. If $\varrho(A / Z)$ is replaced by $\bar{\varrho}(A / Z)$ in (b), then $\bar{\varrho}\left(A \otimes A^{\prime} / Z \otimes Z^{\prime}\right)=\left(Z \otimes Z^{\prime}\right) \times \bar{\varrho}(A / Z)^{w^{\prime}} \otimes \bar{\varrho}\left(A / Z^{\prime}\right)^{w}$.

Proof. (1) By [43, Lemma 1.1], $Z \otimes Z^{\prime}$ is a domain. That $Z \otimes Z^{\prime}$ is CM follows by the same argument as in Theorem 1.14. Since $\mathbb{k}$ is algebraically closed, every simple module over $Z \otimes Z^{\prime}$ is a tensor product of simple modules over $Z$ and $Z^{\prime}$ respectively. Thus, singular points on $\operatorname{Spec} Z \otimes Z^{\prime}$ are of the form $\left(z, z^{\prime}\right)$ where either $z \in \operatorname{Spec} Z$ or $z^{\prime} \in \operatorname{Spec} Z^{\prime}$ is singular. This implies that the singular locus of $\operatorname{Spec} Z \otimes Z^{\prime}$ has codimension $\geq 2$. By Serre's criterion for normality, $Z \otimes Z^{\prime}$ is normal.

(2) It is clear that the center of $A \otimes A^{\prime}$ is $Z \otimes Z^{\prime}$. Since $Z$ is affine and CM, by Noether normalization, there is a polynomial subring $S \subseteq Z$ such that $Z$ is a finite free module over $S$. Since $A$ is CM over $Z, A$ is a finite free module over $S$. Similarly, there is a polynomial subring $S^{\prime} \subseteq Z^{\prime}$ such that both $Z^{\prime}$ and $A^{\prime}$ are finite free module over $S^{\prime}$. Then $A \otimes A^{\prime}$ is finite and free over the central polynomial subring $S \otimes S^{\prime}$. Hence $A \otimes A^{\prime}$ is CM over $S \otimes S^{\prime}$ and over $Z \otimes Z^{\prime}$.

(3) Let $U$ be the non-singular locus of $X:=\operatorname{Spec} Z$. Then $X \backslash U$ has codimension $\geq 2$ in $X$ as $Z$ is normal. Similarly, the non-singular locus of $X^{\prime}:=\operatorname{Spec} Z^{\prime}$, denoted by $U^{\prime}$, has complement with codimension $\geq 2$ in $X^{\prime}$. Since $\mathbb{k}$ is algebraically closed, $U \times U^{\prime}$ is an open scheme of the non-singular locus of $X \times X^{\prime}:=\operatorname{Spec}\left(Z \otimes Z^{\prime}\right)$ whose complement has codimension $\geq 2$ in $X \times X^{\prime}$. By Lemma 2.4(1) and a local version of Lemma 1.12 (with $\mathbf{b}=\mathbf{x}$ and $\left.\mathbf{b}^{\prime}=\mathbf{x}^{\prime}\right)$, over $U \times U^{\prime}, \operatorname{MD}\left(A \otimes A^{\prime} / Z \otimes Z^{\prime}\right.$ ) is equal to $\operatorname{MD}\left(A \otimes A^{\prime} / Z \otimes Z^{\prime}\right)^{\vee \vee}$ and equal to the Cartier divisor $\mathcal{O}_{U \times U^{\prime}}(D)$ where $D$ is determined by $d:=\varrho(A / Z)^{w^{\prime}} \varrho\left(A / Z^{\prime}\right)^{w}$. Since $d$ is defined over $X \times X^{\prime}, \operatorname{MD}\left(A \otimes A^{\prime} / Z \otimes Z^{\prime}\right)^{\vee \vee}$ is the principal ideal generated by $d$ by Lemma 2.2, The assertion follows.

(3') This is similar to the proof of (3).

\section{3. $\mathcal{R}_{v}^{p}$-DISCRIMINANTS}

Next, we introduce the $p$-power reflexive hull $v$-discriminant (or $\mathcal{R}_{v}^{p}$-discriminant), as well as its extended counterpart, the $\overline{\mathcal{R}}_{v}^{p}$-discriminant. These notions generalize the $p$-power discriminants introduced in [18]. As we illustrate in Example 3.2, there are situations in which the $\mathcal{R}$-discriminant does not exist, but for some $p$, the $\mathcal{R}_{v^{-}}^{p}$ discriminants do exist. Hence, in these situations, the $\mathcal{R}_{v^{-}}^{p}$ discriminant can serve as a useful invariant (see Theorem 3.3). For the sake of brevity, in this section, we omit some non-essential details.

Definition 3.1. Assume Hypothesis 1.1 for $(B, R)$. Fix two positive integers $p, v$.

(1) The $p$-power $v$-discriminant ideal, denoted by $\operatorname{MD}_{v}^{p}(B / R)$, is the ideal $\left(\operatorname{MD}_{v}(B / R)\right)^{p}$ (where $\mathrm{MD}_{v}(B / R)$ is given in Definition $\left.1.2(3)\right)$.

(2) The $p$-power reflexive hull $v$-discriminant ideal or $\mathcal{R}_{v}^{p}$-discriminant ideal of $B$ over $R$ is defined to be

$$
\mathcal{R}_{v}^{p}(B / R)=\left(\mathrm{MD}_{v}^{p}(B / R)\right)^{\vee \vee} .
$$

(3) If further $\mathcal{R}_{v}^{p}(B / R)$ is a principal ideal generated by an element $d$ in $R$, then $d$ is called the p-power reflexive hull $v$-discriminant or $\mathcal{R}_{v}^{p}$-discriminant of $B$ over $R$ and denoted by $\varrho_{v}^{[p]}(B / R)$.

(4) The p-power extended reflexive hull $v$-discriminant ideal or $\overline{\mathcal{R}}_{v}^{p}$-discriminant ideal of $B$ over $R$ is defined to be

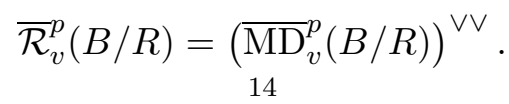


(5) If further $\overline{\mathcal{R}}_{v}^{p}(B / R)$ is a principal ideal generated by a normal element $d$ in $B$, then $d$ is called the p-power extended reflexive hull v-discriminant or $\overline{\mathcal{R}}_{v}^{p}$-discriminant of $B$ over $R$ and denoted by $\varrho_{v}^{[p]}(B / R)$.

It is clear that if it exists, $\varrho_{v}^{[p]}(B / R)\left(\operatorname{resp} . \varrho_{v}^{[p]}(B / R)\right)$ is unique up to a unit in $R$ (resp. in $\left.B\right)$.

Theorem 4.8 in the next section indicates that if $R$ (resp. $B$ ) is nice enough, then $\varrho_{v}^{[p]}(B / R)$ (resp. $\left.\varrho_{v}^{[p]}(B / R)\right)$ exists. In general, the existence of $\varrho_{v}^{[p]}(B / R)$ (resp. $\left.\varrho_{v}^{[p]}(B / R)\right)$ is dependent on $(p, v)$ as the next example shows.

Example 3.2. Let $Z=\mathbb{k}[a, b, c] /\left(a b-c^{3}\right)$ and consider the $A_{2}$ singularity $X=\operatorname{Spec}(Z)$. The divisor class group $\operatorname{Div}(X)$ of $X$ is isomorphic to $\mathbb{Z} / 3 \mathbb{Z}$ with generator given by $I=(a, c)$. Let $A$ be the matrix algebra $\left(\begin{array}{ll}Z & I \\ Z & Z\end{array}\right)$. Then the modified discriminant is $\operatorname{MD}(A / Z)=I^{2}$. The rank of $A$ over its center $Z$ is 4 .

(1) For every $q \geq 1, I^{q}$ is not principal, and so no $p$-power discriminant ideal $\operatorname{MD}(A / Z)^{p}$ is principal.

(2) The $\mathcal{R}$-discriminant ideal $\left(I^{2}\right)^{\vee \vee}$ is not principal as $\operatorname{Div}(X) \cong \mathbb{Z} / 3 \mathbb{Z}$ with generator given by $I=(a, c)$. So $\varrho(A / Z)$ (namely, $\left.\varrho_{4}^{[1]}(A / Z)\right)$ does not exist.

(3) Since $I^{3}=\left(a^{3}, a^{2} c, a c^{2}, c^{3}\right)=a\left(a^{2}, a c, c^{2}, b\right)$ and $\operatorname{GKdim}\left(Z /\left(a^{2}, a c, c^{2}, b\right)\right)=0$ we have, by Lemma 1.5(1), that $\left(I^{3}\right)^{\vee \vee}=(a)$ is principal. Hence $\left(I^{6}\right)^{\vee \vee}=\left(a^{2}\right)$ is also principal. This means that $\varrho_{4}^{[3]}(A / Z)==_{\mathbb{k}} \times a^{2}$.

(4) One can check that

$$
\varrho_{w}^{[p]}(A / Z)= \begin{cases}1 & 1 \leq w \leq 3, \quad p \geq 1 \\ a^{\frac{p}{3}} & w=3, \quad 3 \mid p, \\ \text { does not exist } & w=3 \text { or } w=4, \quad 3 \nmid p, \\ a^{\frac{2 p}{3}} & w=4, \quad 3 \mid p \\ 0 & w \geq 5, \quad p \geq 1 .\end{cases}
$$

The proof of the following result is similar to the proof of Theorem 1.11 and is omitted.

Theorem 3.3. Let $(A, Z)$ satisfy Hypotheses 1.1 and 1.10(1) where $Z$ is the center of $A$. Fix two positive integers $p, v$.

(1) Suppose $d$ is an $\mathcal{R}_{v}^{p}$-discriminant of $A$ over $Z$. If $g \in \operatorname{Aut}(A)$, then $g(d)={ }_{Z \times} d$.

(2) Suppose $d$ is an $\overline{\mathcal{R}}_{v}^{p}$-discriminant of $A$ over $Z$. If $g \in \operatorname{Aut}(A)$, then $g(d)={ }_{A^{\times}} d$.

(3) Let $d$ be either $\varrho(A / Z)$ or $\varrho(A / Z)$. Suppose that char $\mathbb{k}=0$ and that $A^{\times}=\mathbb{k}^{\times}$. If $\delta$ is a locally nilpotent derivation of $A$, then $\delta(d)=0$.

\section{Proof of Theorem 0.2}

In this section we first make a few elementary remarks about Hypotheses 1.1, 1.10, and 2.1 and then prove Theorem 0.2 .

Let $A$ be a prime affine algebra that is module-finite over its center $Z:=Z(A)$. Following Brown and Hajarnavis [10], $A$ is called homologically homogeneous (or hom-hom for short) of dimension $d$ if all simple $A$-modules have the same projective dimension $d$ (see also [48, 49]). Hom-hom rings appear naturally in several contexts. 
Example 4.1. The following are examples of hom-hom rings:

(1) Affine noetherian prime Hopf algebras that are module-finite over their centers and have finite global dimension [9, Theorem A].

(2) Connected graded noetherian Artin-Schelter regular PI algebras [49]. These include the PI skew polynomial rings defined in Definition 0.1 .

(3) Noncommutative crepant resolutions, in the sense of Van den Bergh [51].

The results in the following lemma are well-known.

Lemma 4.2. [10, 48, 49] Let $A$ be a hom-hom ring with center $Z$.

(1) $Z$ is normal. As a consequence, Hypothesis 2.1 holds.

(2) The image of $\operatorname{tr}$ is in $Z$. As a consequence, Hypothesis 1.1 holds.

(3) Suppose $Z$ is affine. Then $A$ is a reflexive module over $Z$.

Proof. (1) This is [10, Theorem 6.1] (or [49, Theorem 5.6(ii)]). Note that a Krull domain is just a noetherian normal domain.

(2) By [49, Theorem 5.4(iii)], $A$ is equal to its trace ring, which implies, by definition, that the image of $\operatorname{tr}$ is in $Z$.

(3) By [48, Theorem 2.3(1,4)], $A$ is a CM tame $Z$-order in the sense of [48, Section 2]. So $A$ is a finitely generated CM (and then free) module over a polynomial subring $R$ of $Z$. Hence $A$ is reflexive over $R$. It follows from [48, Lemma 2.1] that $A$ is also reflexive over $Z$.

Lemma 4.3. Let $A$ be a prime ring with center $Z$. Suppose that $Z$ is normal and that char $\mathbb{k}$ does not divide the rank of $A$ over $Z$. Then $Z$ is CM. As a consequence, Hypothesis 1.10(1) holds.

Proof. If char $\mathbb{k}=0$, then the result follows from [48, Theorem 2.3(3)]. Now suppose that char $\mathbb{k}>$ 0 . Since char $\mathbb{k}$ does not divide the rank of $A$ over $Z$, tr is a nonzero scalar multiple of the identity map when restricted to $Z$. Then $Z$ is a $Z$-module direct summand of $A$, and the assertion follows from [10, Theorem 2.5].

Note that Hypothesis 1.10(1) fails if char $\mathbb{k}$ divides $\operatorname{rk}_{Z}\left(A_{Z}\right)$, see [11, Example 7.3] for a local example. An affine version can be made using the idea of [11, Example 7.3], see explanation in [12, Example 2.3(vi)].

Hypothesis 1.10(2) is a technical condition and we expect that Lemma 1.12 and Theorem 1.14 hold without this hypothesis, see Theorem 2.6.

For a hom-hom ring $A$, the dualizing module $\omega_{A}$ is defined in [48, p.663]. We say $A$ is Calabi-Yau if $\omega_{A} \cong A$ as $A$-bimodule [48, Remark 3.2]. When $A$ is a prime affine algebra and module-finite over its center, this definition is equivalent to other definitions, for example, the one given given by Ginzburg [23].

Lemma 4.4. Let $A$ be an affine Calabi-Yau prime algebra that is module-finite over its center $Z$. Then $Z$ is Gorenstein.

Proof. This follows from [48, Lemma 2.5(5)].

For the rest of this section we prove Theorem 0.2 . The skew polynomial ring $\mathbb{k}_{\mathbf{p}}\left[x_{1}, \ldots, x_{n}\right]$, as in Definition 0.1 , is $\mathbb{Z}^{n}$-graded with $\operatorname{deg} x_{i}=e_{i}$. It is also connected $\mathbb{N}$-graded when we set $\operatorname{deg} x_{i}=1$ for all $i$. 
Lemma 4.5. Let $A$ be the skew polynomial ring $\mathbb{k}_{\mathbf{p}}\left[x_{1}, \ldots, x_{n}\right]$ such that each $p_{i j}$ is a root of unity. Let $r^{2}$ be the rank of $A$ over its center $Z$.

(1) char $\mathbb{k}$ and $r$ are coprime. As a consequence, $Z$ is a CM normal domain.

(2) Let $M$ be an ideal of $A$ generated by monomials, namely, $\mathbb{Z}^{n}$-homogeneous elements. Then $M^{\vee \vee}$ is a principal ideal of $A$.

(3) For every monomial $f, \operatorname{tr}(f)= \begin{cases}r^{2} f & f \in Z \\ 0 & f \notin Z \text {. }\end{cases}$

(4) Let $\mathcal{U}$ and $\mathcal{U}^{\prime}$ be two v-element sets of monomials. Then

$$
d_{v}\left(\mathcal{U}, \mathcal{U}^{\prime}\right) \in Z \cap \mathbb{k} \prod_{f \in \mathcal{U}, f^{\prime} \in \mathcal{U}^{\prime}} f f^{\prime}
$$

which is a monomial in $Z$.

(5) For every pair of positive integers $(p, v), \operatorname{MD}_{v}^{p}(A / Z)$ is generated by a set of monomials in $Z$.

(6) For every pair of positive integers $(p, v), \overline{\mathrm{MD}}_{v}^{p}(A / Z)$ is an ideal of $A$ generated by a set of monomials in $Z$.

Proof. (1) For each $i, j$, let $n_{i j}$ denote the order of $p_{i j}$. If char $\mathbb{k}>0$, then char $\mathbb{k}$ is coprime to $n_{i j}$ for all $i, j$. Since the rank of $A$ over $Z$ is a factor of $\left(\prod_{i<j} n_{i j}\right)^{2}$, it follows that $r$ and char $\mathbb{k}$ are coprime. By Lemma 4.3, $Z$ is CM and by Lemma 4.2(1), $Z$ is normal.

(2) Let $M$ be an ideal of $A$ generated by a set $\mathcal{S}$ of monomials. We can write $M=\mathcal{S} A$ since each element in $\mathcal{S}$ is normal in $A$. For each $i$, there is a positive integer $w_{i}$ such that $a_{i}:=x_{i}^{w_{i}}$ is in $Z$. Let $U_{i}$ denote the open subset of $X:=\operatorname{Spec} Z$ with $\prod_{j \neq i} a_{j} \neq 0$.

Let $U:=\bigcup_{i=1}^{n} U_{i}$. First, we claim that $X \backslash U$ has codimension $\geq 2$. Note that the subalgebra $R=\mathbb{k}\left[a_{1}, \ldots, a_{n}\right]$ is a polynomial subring of $Z$ such that $A$ is finitely generated over $R$. Since $A$ and $Z$ are CM, both $A$ and $Z$ are finitely generated free $R$-modules. As a consequence, $\left\{a_{1}, \ldots, a_{n}\right\}$ is an $R$-regular sequence (and also a $Z$-regular sequence) in $A$ and in $Z$. Write $Z$ as a factor ring $\mathbb{k}\left[a_{1}, \ldots, a_{n}, b_{1}, \ldots, b_{w}\right] / I$ for some generators $b_{1}, \ldots, b_{w}$. If $\mathfrak{m}$ is a closed point which is not in $U$, then there are at least two $i_{0} \neq j_{0}$ such that $a_{i_{0}}=0=a_{j_{0}}$. Since $\left\{a_{1}, \ldots, a_{n}\right\}$ is a $Z$-regular sequence, $\operatorname{GKdim}\left(Z /\left(a_{i_{0}}, a_{j_{0}}\right)\right)=\operatorname{GKdim} Z-2=n-2$. Therefore $X \backslash U$ has dimension at most $n-2$ and we have proved the claim.

Now for each $f \in \mathcal{S}$, write $f=x_{1}^{h_{1}} \cdots x_{n}^{h_{n}}$ and define $p_{i}(f):=h_{i}$ for each $i$. Let $s_{i}=\min \left\{p_{i}(f) \mid\right.$ $f \in \mathcal{S}\}$ and define $d=x_{1}^{s_{1}} \cdots x_{n}^{s_{n}}$. It is clear that $d$ is the gcd of the elements in $\mathcal{S}$ and that $M \subseteq d A$. We now claim that $M^{\vee \vee}$ is the principal ideal of $A$ generated by $d$. Since $A$ is $Z$ reflexive [Lemma 4.2(3)], $d A$ is reflexive and contains $M^{\vee \vee}$. By Lemma 2.2, it suffices to show that $\left.\left(M^{\vee \vee}\right)\right|_{U}=\left.(d A)\right|_{U}$, or equivalently, that for each $i$, we have $\left.\left(M^{\vee \vee}\right)\right|_{U_{i}}=\left.(d A)\right|_{U_{i}}$. Over $U_{i}$, we invert the elements $a_{j}=x^{w_{j}}$ for all $j \neq i$. Note that

$$
\left.A\right|_{U_{i}}=A\left[a_{1}^{-1}, \ldots, \widehat{a_{i}^{-1}}, \ldots, a_{n}^{-1}\right]=A\left[x_{1}^{-1}, \ldots, \widehat{x_{i}^{-1}}, \ldots, x_{n}^{-1}\right]=\mathbb{k}_{\mathbf{p}}\left[x_{1}^{ \pm 1}, \ldots, x_{i-1}^{ \pm 1}, x_{i}, x_{i+1}^{ \pm 1}, \ldots, x_{n}^{ \pm 1}\right] .
$$

Hence

$$
\left.M\right|_{U_{i}}=\left.(\mathcal{S} A)\right|_{U_{i}}=\left.\sum_{f \in \mathcal{S}} f A\right|_{U_{i}}=\left.\sum_{f \in \mathcal{S}} x_{i}^{p_{i}(f)} A\right|_{U_{i}}=\left.x_{i}^{s_{i}} A\right|_{U_{i}}=\left.(d A)\right|_{U_{i}}
$$

which implies that $\left.\left(M^{\vee \vee}\right)\right|_{U_{i}}=\left.(d A)\right|_{U_{i}}$ as required. This proves the claim. 
(3) Consider $A$ as a $\mathbb{Z}^{n}$-graded algebra. Choose a semi-basis of $A$ over $Z$ to consist of $\mathbb{Z}^{n_{-}}$ homogeneous elements (namely, monomials). By linear algebra, for each $\mathbb{Z}^{n}$-homogeneous element $f, \operatorname{tr}(f)$ is either 0 or equal to $r^{2} f$. The second case happens if and only if $f$ is in $Z$.

(4) This is [15, Lemma 2.6].

$(5,6)$ We can choose a generating set of $A$ over $Z$ consisting of $\mathbb{Z}^{n}$-homogeneous elements. Both assertions follow from part (4).

Theorem 4.6. Let $A$ be a skew polynomial ring $\mathbb{k}_{\mathbf{p}}\left[x_{1}, \ldots, x_{n}\right]$ where each $p_{i j}$ is a root of unity. Then, for any two positive integers $p, v, \varrho_{v}^{[p]}(A / Z)$ exists.

Proof. By Lemma 4.5(6), $\overline{\operatorname{MD}}_{v}^{p}(A / Z)$ is generated by a set of monomials. Now the assertion follows from Lemma 4.5(2).

By the proof of Theorem 4.6, one can verify that in this case, $\bar{\varrho}(A / Z)$ agrees with the usual discriminant $d_{r^{2}}(A / Z)$ defined in [15, Definition 1.2(3)].

In Example 2.5, we computed the $\mathcal{R}$ - and $\overline{\mathcal{R}}$-discriminants for several specific skew polynomial rings $\mathbb{k}_{\mathbf{p}}\left[x_{1}, x_{2}, x_{3}\right]$ over their centers. We ask the following question.

Question 4.7. Let $A$ be a skew polynomial ring $\mathbb{k}_{\mathbf{p}}\left[x_{1}, \ldots, x_{n}\right]$ where each $p_{i j}$ is a root of unity. When does the $\mathcal{R}$-discriminant $\varrho(A / Z)$ exist? In terms of $\mathbf{p}$, what are the formulas for $\bar{\varrho}(A / Z)$ and $\varrho(A / Z) ?$

A ring $R$ is called a noetherian unique factorization ring (noetherian UFR) if $R$ is a prime left and right noetherian ring such that every nonzero prime ideal of $R$ contains a non-zero principal prime ideal. Hence, in a noetherian UFR every height one prime ideal is principal. Since the skew polynomial rings in Theorem 4.6 are noetherian hom-hom UFRs, part (2) of the following result is more general than Theorem 4.6, but has a less constructive proof.

Theorem 4.8. Assume Hypothesis 1.1 for $(A, Z)$. Fix two positive integers $p, v$.

(1) If $Z$ is a UFD, then the $\mathcal{R}_{v}^{p}$-discriminant $\varrho_{v}^{[p]}(A / Z)$ exists.

(2) Suppose $A$ is a noetherian hom-hom UFR with affine center $Z$. Then the $\overline{\mathcal{R}}_{v}^{p}$-discriminant $\bar{\varrho}_{v}^{[p]}(A / Z)$ always exists.

Proof. (1) This follows immediately since every reflexive ideal of a UFD is principal.

(2) Since $A$ is hom-hom, by Lemma 4.2(3), $A$ is a tame $Z$-order and a reflexive $Z$-module. Thus, by [48, Lemma 2.1], every reflexive $Z$-module is reflexive as an $A$-module (see also [44, Corollary 1.6]). By [1, Lemma 3.3(ii)], every reflexive ideal of $A$ is principal. It follows that the $\overline{\mathcal{R}}$-discriminant of $A$ over its center exists.

Motivated by the above results, we ask the following questions.

Question 4.9. Let $A$ be a hom-hom ring with center $Z$. Under what conditions does $\bar{\varrho}(A / Z)$ (resp. $\varrho(A / Z))$ exist? For example, if $A$ is Calabi-Yau, does $\varrho(A / Z)$ (resp. $\varrho(A / Z)$ ) exist?

\section{EXAMPLES}

Much of the recent literature on discriminants (e.g., 14, 15, 18, 17]) has focused on connected graded hom-hom rings (namely, noetherian connected graded Artin-Schelter regular algebras satisfying a polynomial identity). In this paper we will test discriminant theory for a class of noncommutative algebras, the quantum generalized Weyl algebras (quantum GWAs), which are generally neither connected graded nor of finite global dimension. 
We begin by introducing GWAs in full generality and recalling some well-known facts regarding GWAs. We then proceed to work out the $\mathcal{R}$-discriminant for quantum GWAs.

5.1. Properties of quantum GWAs. The following definition is motivated by Bavula [2].

Definition 5.1. Let $R$ be a $\mathbb{k}$-algebra, $\sigma=\left(\sigma_{1}, \ldots, \sigma_{m}\right)$ a set of $m$ commuting automorphisms of $R$, and $h=\left(h_{1}, \ldots, h_{m}\right)$ a set of $m$ central regular elements in $R$ such that $\sigma_{i}\left(h_{j}\right)=h_{j}$ for $i, j \in\{1, \ldots, m\}, i \neq j$. With this data, we define the $G W A$ of degree $m$ as the $\mathbb{k}$-algebra generated over $R$ as an algebra by $\mathbf{x}=\left(x_{1}, \ldots, x_{m}\right)$ and $\mathbf{y}=\left(y_{1}, \ldots, y_{m}\right)$ with relations

$$
\begin{array}{llr}
x_{i} r=\sigma_{i}(r) x_{i} & y_{i} r=\sigma_{i}^{-1}(r) y_{i} & \text { for all } i \in\{1, \ldots, m\}, r \in R \\
x_{i} y_{i}=h_{i} & y_{i} x_{i}=\sigma_{i}^{-1}\left(h_{i}\right) & \text { for all } i \in\{1, \ldots, m\} \\
{\left[x_{i}, x_{j}\right]=\left[y_{i}, y_{j}\right]=\left[x_{i}, y_{j}\right]=0} & & \text { for all distinct } i, j \in\{1, \ldots, m\} .
\end{array}
$$

We denote this algebra by $R(\mathbf{x}, \mathbf{y}, \sigma, h)$. We say $R(\mathbf{x}, \mathbf{y}, \sigma, h)$ is a quantum $G W A$ if $R=\mathbb{k}[t]$ and there exist nonzero scalars $q=\left(q_{1}, \ldots, q_{m}\right) \in(\mathbb{k} \backslash\{0,1\})^{m}$ such that $\sigma_{i}(t)=q_{i}$ t for all $i \in\{1, \ldots, m\}$. If $q_{i}$ has finite order in the multiplicative group $\mathbb{k}^{\times}$, then we let $\operatorname{ord}\left(q_{i}\right)$ denote the order of $q_{i}$.

In general, the base ring $R$ in Definition 5.1 need not be commutative but in this paper, $R$ is always taken to be a commutative polynomial ring. We also only consider the case where each $q_{i}$ is a root of unity. More specifically, we will only consider the case in which the ord $\left(q_{i}\right)$ are pairwise relatively prime.

If $W$ is a GWA of degree $m$, then for each $i=1, \ldots, m$ the subalgebra $W_{i}$ generated by $x_{i}$ and $y_{i}$ is a degree one GWA isomorphic to $R\left(x_{i}, y_{i}, \sigma_{i}, h_{i}\right)$ [Definition 0.5. More generally, let $I \subseteq\{1, \ldots, m\}$. Set $\mathbf{x}_{I}=\left(x_{i}\right)_{i \in I}, \mathbf{y}_{I}=\left(y_{i}\right)_{i \in I}, \sigma_{I}=\left(\sigma_{i}\right)_{i \in I}$, and $h_{I}=\left(h_{i}\right)_{i \in I}$. Then $\left\{\mathbf{x}_{I}, \mathbf{y}_{I}\right\}$ generate a degree $|I|$ GWA over $R$ that we denote by $R\left(\mathbf{x}_{I}, \mathbf{y}_{I}, \sigma_{I}, h_{I}\right)$.

The class of GWAs is also closed under tensor products over $\mathbb{k}$. If $W=R(\mathbf{x}, \mathbf{y}, \sigma, h)$ and $W^{\prime}=R^{\prime}\left(\mathbf{x}^{\prime}, \mathbf{y}^{\prime}, \sigma^{\prime}, h^{\prime}\right)$ are GWAs of degree $k$ and $\ell$, respectively, then

$$
W \otimes W^{\prime}=\left(R \otimes R^{\prime}\right)\left(\left(\mathbf{x}, \mathbf{x}^{\prime}\right),\left(\mathbf{y}, \mathbf{y}^{\prime}\right),\left(\sigma, \sigma^{\prime}\right),\left(h, h^{\prime}\right)\right)
$$

is a GWA of degree $k+\ell$. The following facts are well-known.

Lemma 5.2. Let $R$ be a $\mathbb{k}$-algebra and suppose $W=R(\mathbf{x}, \mathbf{y}, \sigma, h)$ is a degree $m G W A$ over $R$.

(1) If $R$ is a left (right) noetherian ring, then so is $W$.

(2) If $R$ is a domain, then so is $W$.

(3) There is a $\mathbb{Z}^{m}$-grading on $W$ obtained by setting $\operatorname{deg}(r)=\mathbf{0}$ for all $r \in R$ and $\operatorname{deg}\left(x_{i}\right)=\mathbf{e}_{i}$, $\operatorname{deg}\left(y_{i}\right)=-\mathbf{e}_{i}$ for all $i$.

Suppose $W=R(\mathbf{x}, \mathbf{y}, \sigma, h)$ is a GWA of degree $m$. For $\gamma=\left(\gamma_{1}, \ldots, \gamma_{m}\right) \in \mathbb{Z}^{m}$, let $z^{\gamma}$ denote $z_{1}^{\gamma_{1}} \ldots z_{m}^{\gamma_{m}}$ formally where $z_{i}^{\gamma_{i}}=x_{i}^{\gamma_{i}}$ when $\gamma_{i} \geq 0$ and $z_{i}^{\gamma_{i}}=y_{i}^{-\gamma_{i}}$ when $\gamma_{i}<0$. By a result of Benkart and Ondrus [7, Proposition 2.5], the center $Z(W)$ of $W$ is generated by $R^{\langle\sigma\rangle}=\{r \in R \mid$ $\sigma_{i}(r)=r$ for all $\left.i=1, \ldots, m\right\}$ and those monomials $z^{\gamma}$ such that $\sigma^{\gamma}:=\prod_{i=0}^{m} \sigma_{i}^{\gamma_{i}}=\operatorname{Id}_{R}$. Note that this generalizes a result of Kulkarni [31, Corollary 2.0.2] in the case of a degree one GWA over a commutative domain.

In subsection 5.3 we will use the following.

Corollary 5.3. Let $R$ a commutative domain and let $W=R(\mathbf{x}, \mathbf{y}, \sigma, h)$ be a $G W A$ of degree $m$ with $n_{i}=\left|\sigma_{i}\right|<\infty$ for all $i$. Suppose $\operatorname{gcd}\left(n_{i}, n_{j}\right)=1$ for all $i \neq j$. Then $Z(W)$ is generated over $R^{\langle\sigma\rangle}$ by $x_{1}^{n_{1}}, y_{1}^{n_{1}}, \ldots, x_{m}^{n_{m}}, y_{m}^{n_{m}}$. 
Proof. By the hypotheses on the $n_{i}$, the subgroup of $\operatorname{Aut}(R)$ generated by the $\sigma_{i}$ is a finite abelian group of order $n=\prod_{i=1}^{m} n_{i}$. The result now follows directly from [7, Proposition 2.5].

An automorphism $\sigma$ of a $\mathbb{k}$-algebra $R$ is called locally algebraic if every finite-dimensional subspace of $R$ is contained in a finite-dimensional $\sigma$-stable subspace of $R$. It is clear that if $\sigma$ has finite order, then $\sigma$ is locally algebraic.

The following result uses [19] but it may also be possible to apply [54 to the same effect.

Lemma 5.4. Let $R$ be an affine $\mathbb{k}$-algebra and $W:=R(\mathbf{x}, \mathbf{y}, \sigma, h)$ a degree $m G W A$ over $R$. If $\left|\sigma_{i}\right|<\infty$ for all $i$, then $\operatorname{GKdim} W=\operatorname{GKdim} R+m$.

Proof. Let $W(1)=R\left(x_{1}, y_{1}, \sigma_{1}, h_{1}\right)$. Since $\left|\sigma_{1}\right|<\infty$, then $\sigma_{1}$ is locally algebraic. Hence, [19, Theorem 26] gives GKdim $W(1)=\operatorname{GKdim} R+1$.

Let $k \geq 1, I=\{1, \ldots, k\}$, and $W(k)=R\left(\mathbf{x}_{I}, \mathbf{y}_{I}, \sigma_{I}, h_{I}\right)$. Assume that GKdim $W(k)=$ GKdim $R+k$. Recall that $W(k+1) \cong W(k)\left(x_{k+1}, y_{k+1}, \sigma_{k+1}, h_{k+1}\right)$ where $\sigma_{k+1}$ extends to $W(k)$ by setting $\sigma_{k+1}\left(x_{i}\right)=x_{i}$ and $\sigma_{k+1}\left(y_{i}\right)=y_{i}$ for all $i<k+1$. Consequently, $\left|\sigma_{k+1}\right|$ has finite order as an automorphism of $W(k)$ and so $\sigma_{k+1}$ is locally algebraic on $W(k)$. Applying [19, Theorem 26] again gives

$$
\operatorname{GKdim} W(k+1)=\operatorname{GKdim} W(k)+1=\operatorname{GKdim} R+(k+1) .
$$

Now the assertion follows by induction.

Conjecture 5.5. The conclusion of the previous lemma holds whenever $R$ is a finitely generated module over $R^{G}$ where $G=\left\langle\sigma_{1}, \ldots, \sigma_{m}\right\rangle$.

The following is an extended version of [29, Proposition 3.2]. We refer the reader there for the definition of Auslander-Gorenstein.

Lemma 5.6. Let $W=\mathbb{k}[t](\mathbf{x}, \mathbf{y}, \sigma, h)$ be a $G W A$ of degree $m$. Then $W$ is Auslander-Gorenstein and GK-Macaulay.

Proof. Set $S_{0}=\mathbb{k}[t]$. For $i=1, \ldots, m$, let $S_{i}=S_{i-1}\left[x_{i} ; \sigma_{i}\right]$ where $\sigma_{i}$ is extended to $S_{i-1}$ by setting $\sigma_{i}\left(x_{j}\right)=x_{j}$ for $j<i$. Set $T_{0}=S_{m}$. For $i=1, \ldots, m$, let $T_{i}=T_{i-1}\left[y_{i} ; \sigma_{i}^{-1}, \delta_{i}\right]$ where $\sigma_{i}^{-1}$ is extended to $T_{i-1}$ by setting $\sigma_{i}^{-1}\left(y_{j}\right)=y_{j}$ for $j<i, \delta_{i}(r)=0$ for all $r \in R, \delta_{i}\left(y_{j}\right)=\delta_{i}\left(x_{j}\right)=0$ for $i \neq j$, and $\delta_{i}\left(x_{i}\right)=\sigma_{i}^{-1}\left(h_{i}\right)-h_{i}$. Set $T=T_{m}$.

Let $k_{i}=\operatorname{deg}_{t}\left(h_{i}\right)$. Filter $T$ by setting $\operatorname{deg}(t)=2$ and $\operatorname{deg}\left(x_{i}\right)=\operatorname{deg}\left(y_{i}\right)=k_{i}$. Then $\operatorname{gr}(T)$ is a connected graded ring which itself is an iterated Ore extension over $\mathbb{k}[t]$. For each $i=1, \ldots, m$, $\bar{\sigma}_{i}$ preserves the grading on $\operatorname{gr}(T)$ so $\operatorname{gr}(T)$ is GK-Macaulay by [32, Lemma (ii)]. Thus, $T$ is Auslander-Gorenstein and GK-Macaulay by [49, Lemma 4.4].

Since $\sigma_{i}\left(h_{j}\right)=h_{j}$ for $i \neq j$, then the elements $z_{i}=x_{i} y_{i}-h_{i}$ are central in $T$. Moreover, each $W_{i}$ is a domain and so $\left(z_{1}, \ldots, z_{m}\right)$ is a regular sequence of central elements. Hence, $T /\left(z_{1}, \ldots, z_{m}\right) \cong W$ is Auslander-Gorenstein and GK-Macaulay by [32, Lemma (iii)].

The global dimension of degree one GWAs has been studied extensively. Less is known in the case of higher degree GWAs. For our purposes, the next result is sufficient.

Lemma 5.7 ([3, 27]). Let $W=\mathbb{k}[t](x, y, \sigma, h)$ be a quantum $G W A$ with $q \neq 1$ a root of unity. If $h$ has multiple roots, then gldim $W=\infty$. Otherwise, gldim $W=2$. 
5.2. The $\mathcal{R}$-discriminant of a degree one quantum GWA. In this subsection, let $W=$ $\mathbb{k}[t](x, y, \sigma, h)$ denote a degree one quantum GWA with $n=|\sigma|<\infty$. We study the center of $W$ and the trace map, and conclude this subsection by computing the reflexive hull discriminant of $W$ over its center. This subsection serves as a warmup to the next subsection where we study higher degree quantum GWAs, but omit some details.

By [31, Corollary 2.0.2], the center of $W$ is generated by $a=x^{n}, b=y^{n}, c=t^{n}$. Set $p(c)=$ $\prod_{j=0}^{n-1} h^{\sigma^{j}}(t)$ where $h^{\sigma^{j}}(t)=h\left(\sigma^{j}(t)\right)=h\left(q^{j} t\right)$. Then the generators satisfy a single relation as follows

$$
Z:=Z(W)=\mathbb{k}[a, b, c] /(a b-p(c)) .
$$

Lemma 5.8. The center $Z$ given in (E5.7.1) is an affine normal CM domain. As a consequence, Hypotheses 1.10(1) and 2.1] hold for $(W, Z)$.

Proof. By definition, $Z$ has hypersurface singularities and so it is Gorenstein (and consequently, $\mathrm{CM}$ ). It is clear that $Z$ has isolated singularities. Hence $Z$ is normal by Serre's criterion for normality.

It is easy to see that the rank of $W$ over $Z$ is $n^{2}$. Since $q$ is a primitive $n$th root of unity, char $\mathbb{k}$ does not divide $\operatorname{rk}_{Z}\left(W_{Z}\right)$. The consequence follows from [40, Theorem 10.1] and Lemma 4.3.

Note that the form of the trace map tr is not essential in our proof of the main result of this section [Theorem 5.11]. However, a proof of Theorem 5.11 can be done totally algebraically, in which case the trace map would be used in an essential way. In this particular case, it is easy to work out the trace map. We can write $W$ as a $Z$-algebra as follows

$$
W=\frac{Z\langle x, y, t\rangle}{\left(x t-q t x, y t-q^{-1} t y, x y-h(t), x^{n}-a, y^{n}-b, t^{n}-c\right)} .
$$

Using this presentation, observe that $W$ is generated as a $Z$-module by the following elements

$$
\left\{x^{i} t^{j}, y^{i} t^{j} \mid i, j=0, \ldots, n-1\right\} \text {. }
$$

Let $\tau=\left(1, t, t^{2}, \ldots, t^{n-1}\right) \in W^{\oplus n}$ and

$$
\mathbf{v}=\left(\tau, x \tau, x^{2} \tau, \ldots, x^{n-1} \tau, y \tau, y^{2} \tau, \ldots, y^{n-1} \tau\right) \in W^{\oplus\left(2 n^{2}-n\right)},
$$

considered as a column vector (or a $\left(2 n^{2}-n\right) \times 1$ matrix). The components of $\mathbf{v}$ generate $W$ as a module over $Z$ (E5.8.2), and the first $n^{2}$ components of $\mathbf{v}$ form a semi-basis of $W$ (with respect to $\mathbf{v})$. Consequently, the rank of $W$ over $Z$ is $n^{2}$. The modified discriminant ideal $\operatorname{MD}(W / Z)=$ $\mathrm{MD}_{n^{2}}(W / Z)$ of $W$ is generated by the $n^{2} \times n^{2}$ minors of the $\left(2 n^{2}-n\right) \times\left(2 n^{2}-n\right)$ matrix

$$
D=\operatorname{tr} \cdot \mathbf{v} \mathbf{v}^{T} .
$$

We show that most of the entries of $D$ are zero in the next lemma.

Lemma 5.9. We have $\operatorname{tr}\left(x^{i} t^{j}\right) \neq 0$ and $\operatorname{tr}\left(y^{i} t^{j}\right) \neq 0$ if and only if $i \equiv 0 \bmod n$ and $j \equiv 0 \bmod n$.

Proof. The $Z$-algebra $W$ has a $\mathbb{Z} / n \mathbb{Z}$-graded algebra structure, obtained by assigning the $Z$-algebra generators $x, y, t$ the degrees $1,-1,0$ respectively. Indeed, the relations provided in (E5.8.1) are all homogeneous under this grading.

Left multiplication by $x^{i} t^{j}$ shifts the degree by a nonzero integer unless $i \equiv 0 \bmod n$. Therefore, if $i \not \equiv 0 \bmod n$, then $\operatorname{tr}\left(x^{i} t^{j}\right)=0$. Next assume $i=k n$ for some integer $k$. Left multiplication by $x^{k n} t^{j}$ for $j \not \equiv 0 \bmod n$ permutes the semi-basis $\left\{x^{i} t^{j}\right\}_{i, j=0}^{n-1}$ (up to scalar). Therefore, if $i, j \not \equiv 0 \bmod$ 
$n$, then $\operatorname{tr}\left(x^{i} t^{j}\right)=0$. Finally, if $i \equiv j \equiv 0 \bmod n$, then $x^{i} t^{j} \in Z$ so left multiplication by $x^{i} t^{j}$ can be represented by a diagonal matrix, hence $\operatorname{tr}\left(x^{i} t^{j}\right)=n^{2} x^{i} t^{j}$. The above argument still works if we replace all occurrences of $x$ by $y$. So this completes the proof.

It is very complicated to compute the $\mathcal{R}$-discriminant by using the definition, since $\operatorname{MD}(W / Z)$ is complicated. So we will compute the $\mathcal{R}$-discriminant locally first. In the following lemma, we use the elements of $\mathbf{v}$ to index the rows and columns of $D$, so that the first row of $D$ is the 1 row, the second row is the $t$ row, and so on.

Lemma 5.10. The following hold for the $\left(2 n^{2}-n\right) \times\left(2 n^{2}-n\right)$ square matrix $D=\operatorname{tr} \cdot \mathbf{v v} \mathbf{v}^{T}$.

(1) Let $0<i<n$. The only nonzero entries in the $x^{i} t^{j}$ row of $D$ are in the $x^{n-i} t^{n-j}$ and $y^{i} t^{j^{\prime}}$ columns for some $0 \leq j^{\prime} \leq n-1$.

(2) The statement in (1) holds if we switch $x$ and $y$.

(3) The only nonzero entry in row $t^{j}$ is in column $t^{n-j}$.

Proof. Consider the product $\rho=\left(x^{i} t^{j}\right)\left(y^{k} t^{l}\right)$. Using Lemma 5.9, and the grading on $W$ given in the proof, we see that $\operatorname{tr}(\rho) \neq 0$ implies that $i \equiv k \bmod n$. Similarly, $\operatorname{tr}\left(\left(x^{i} t^{j}\right)\left(x^{k} t^{l}\right)\right) \neq 0$ if and only if $i+k \equiv 0 \bmod n$ and $j+l \equiv 0 \bmod n$. The statement for $\operatorname{tr}\left(t^{j}\right)$ follows similarly.

Recall from Lemma 5.8 that $Z$ is a normal CM domain.

Theorem 5.11. Retain the above notation. Then $\varrho(W / Z)={ }_{Z^{\times}} c^{n(n-1)}={ }_{Z^{\times}} t^{n^{2}(n-1)}$. As a consequence, $\bar{\varrho}(W / Z)={ }_{W} \times c^{n(n-1)}=_{W \times} t^{n^{2}(n-1)}$.

Proof. Let $U_{a}$ and $U_{b}$ denote the open subsets of $X$ where $a \neq 0$ and $b \neq 0$ respectively. Note that the complement of $U:=U_{a} \cup U_{b}$ has codimension 2. It is easy to check that $W_{a}:=\left.W\right|_{U_{a}}=W\left[a^{-1}\right]$ is free of rank $n^{2}$ and has basis $\left\{x^{i} t^{j} \mid i, j=0, \ldots, n-1\right\}$. By [17, Proposition 1.4(2)] or by a computation using Lemma $5.10(1,3)$, the discriminant of $W_{a}$ is given by $(a c)^{n(n-1)}={ }_{Z\left(W_{a}\right) \times} c^{n(n-1)}$, up to some unit in $Z\left(W_{a}\right)$. Similarly the discriminant of $W_{b}$ is given by $(b c)^{n(n-1)}={ }_{Z\left(W_{b}\right) \times} c^{n(n-1)}$, again up to some unit in $Z\left(W_{b}\right)$. This defines a Cartier divisor on $U$, which extends to a Cartier divisor on all of $X$. Indeed, the data $\left\{\left(U_{a}, c^{n(n-1)}\right),\left(U_{b}, c^{n(n-1)}\right),\left(X, c^{n(n-1)}\right)\right\}$ defines a Cartier divisor on $X$ which restricts to the above Cartier divisor on $U$. The assertion now follows by Lemma 2.2 .

For the consequence, we must show that $W$ is a reflexive $Z$-module. By Lemma 5.6, $W$ is GKMacaulay, or equivalently, $W$ is CM. In particular, $W$ satisfies Serre's S2 property. Since $\operatorname{Spec}(Z)$ is a normal surface, it satisfies the hypotheses of [24, Proposition 1.9], so we conclude that $W$ is reflexive.

5.3. The $\mathcal{R}$-discriminant of a degree $m$ quantum GWA. Our goal in this subsection is to generalize results from the previous subsection to higher degree quantum GWAs. This result will be used in our applications.

Throughout this subsection, let $W=\mathbb{k}[t](\mathbf{x}, \mathbf{y}, \sigma, h)$ be a quantum GWA of degree $m$. As in Corollary 5.3. set $n_{i}=\left|\sigma_{i}\right|<\infty$ for all $i$ and assume $\operatorname{gcd}\left(n_{i}, n_{j}\right)=1$ for all $i \neq j$. Set $n=n_{1} \cdots n_{m}$. The center $Z:=Z(W)$ of $W$ is generated by $a_{i}=x_{i}^{n_{i}}, b_{i}=y_{i}^{n_{i}}$, and $c=t^{n}$. Let

$$
p_{i}(c)=\prod_{\substack{j=0 \\ 22}}^{n_{i}-1} h_{i}^{\sigma_{i}^{j}}(t)
$$


where $h_{i}^{\sigma_{i}^{j}}(t)=h_{i}\left(\sigma_{i}^{j}(t)\right)=h_{i}\left(q_{i}^{j} t\right)$. Thus, by Corollary 5.3,

$$
Z=\frac{\mathbb{k}\left[a_{1}, \ldots, a_{m}, b_{1}, \ldots, b_{m}, c\right]}{\left(a_{i} b_{i}-p_{i}(c) \mid 1 \leq i \leq m\right)} .
$$

In Theorem 5.14 we show that the $\mathcal{R}$-discriminant of $W$ is $c^{n(n-1)} \in Z$.

Lemma 5.12. Let $Z$ be the algebra defined as above. Then $Z$ is an affine normal CM domain. As a consequence, Hypotheses 1.10(1) and 2.1 hold for $(W, Z)$.

Proof. By definition, $Z$ is a complete intersection. Hence it is Gorenstein and CM. By the Jacobian criterion, the singular locus of $X:=\operatorname{Spec} Z$ has codimension $\geq 2$. Indeed, the Jacobian matrix of the defining ideal is given by the block matrix $J=\left(\operatorname{diag}\left(b_{1}, \cdots, b_{m}\right)\left|\operatorname{diag}\left(a_{1}, \cdots, a_{m}\right)\right| v\right)$ where $v$ is $m \times 1$ and depends only on $c$. The singular locus of $X$ occurs when $\operatorname{rank}(J)<m$, and a necessary condition for this is $a_{i}=b_{i}=0$ for some $i$. This shows that $\operatorname{codim}_{X}\left(X_{\operatorname{sing}}\right) \geq 2$, so $X$ is normal by Serre's criterion for normality.

We can write $W$ as a $Z$-algebra as follows:

$$
W=\frac{Z\left\langle x_{1} \ldots, x_{m}, y_{1}, \ldots, y_{m}, t\right\rangle}{\left(x_{i} t-q_{i} t x_{i}, y_{i} t-q_{i}^{-1} t y_{i}, x_{i} y_{i}-h_{i}(t), x_{i}^{n_{i}}-a_{i}, y_{i}^{n_{i}}-b_{i}, t^{n}-c\right)} .
$$

Lemma 5.13. Retain the above notation. Then $W$ is reflexive over $Z$.

Proof. The proof is almost identical to the dimension 2 case (c.f. Theorem 5.11). Since $Z$ is a CM with $\operatorname{codim}_{X}\left(X_{\text {sing }}\right) \geq 2$ (where $X:=\operatorname{Spec} Z$ ), the hypotheses of [24, Proposition 1.9] are satisfied. Then $W$ is $Z$-reflexive since it is a CM [Lemma 5.6] (hence S2) $Z$-module.

Here is the main result of this subsection.

Theorem 5.14. Let $W$ be a quantum GWA of degree $m>1$ as given in (E5.12.1). Then we have $\varrho(W / Z)={ }_{Z \times} c^{n(n-1)}={ }_{Z \times} t^{n^{2}(n-1)}$. As a consequence, $\bar{\varrho}(W / Z)={ }_{W \times} c^{n(n-1)}=_{W \times} t^{n^{2}(n-1)}$.

Proof. Let $U$ denote the open set of $X:=\operatorname{Spec} Z$ with $\prod_{i=1}^{m} a_{i} \neq 0$. Then $\left.W\right|_{U}$ is free over its center, with basis given by $\left\{\mathbf{x}^{\alpha} t^{j}:=x_{1}^{\alpha_{1}} x_{2}^{\alpha_{2}} \cdots x_{m}^{\alpha_{m}} t^{j}\right\}$ where $\alpha_{i}$ ranges from 0 to $n_{i}-1$ and $j$ ranges from 0 to $n-1$ (here $n=n_{1} \cdots n_{m}$ ). This shows that $\operatorname{rk}\left(\left.W\right|_{U}\right)=n^{2}$.

We can use (E5.12.1) to compute the discriminant of $\left.W\right|_{U}$. Given a basis element $b=\mathbf{x}^{\alpha} t^{j}$, there is exactly one other basis element $b^{\prime}$ whose product with $b$ has nonzero trace. Moreover $b^{\prime}$ is given as follows

$$
b^{\prime}= \begin{cases}1 & \text { if } \alpha=0, j=0 \\ t^{n-j} & \text { if } \alpha=0, j \neq 0 \\ \mathbf{x}^{\alpha^{\prime}} & \text { if } \alpha \neq 0, j=0 \\ \mathbf{x}^{\alpha^{\prime}} t^{n-j} & \text { otherwise }\end{cases}
$$

where

$$
\alpha_{j}^{\prime}= \begin{cases}0 & \text { if } \alpha_{j}=0 \\ n_{j}-\alpha_{j} & \text { otherwise. }\end{cases}
$$

This shows that the discriminant $d_{U}$ of $\left.W\right|_{U}$ is given by

$$
d_{U}=c^{n(n-1)} \prod_{j=1}^{m} a_{j}^{n\left(n-n / n_{j}\right)} .
$$


That is, $d_{U}={ }_{\left(\left.W\right|_{U}\right) \times} c^{n(n-1)}$. This can be obtained from a direct calculation.

Now consider another open subset $V$ of $X$ where we replace the condition $\prod_{i=1}^{m} a_{i} \neq 0$ with $\prod_{i=1}^{m} a_{i}^{\prime} \neq 0$ where $a_{i}^{\prime}$ is either $a_{i}$ or $b_{i}$. By the symmetry of $\left(x_{i}, y_{i}\right), V$ is another $U$ after we switch some $x_{i}$ with $y_{i}$. Then $d_{V}$ can be obtained by the same computation (by replacing the $a_{i}$ 's with $a_{i}^{\prime}$ 's). Hence $d_{V}={ }_{\left(\left.W\right|_{V}\right)^{\times}} c^{n(n-1)}$. Thus the data $\left\{\left(V, d_{V}:=c^{n(n-1)}\right)\right\}$ (where $V$ ranges over all open subsets with $\prod_{i=1}^{m} a_{i}^{\prime} \neq 0$ ) define a Cartier divisor on $X \backslash C$, where $C$ represents the union of subvarieties $\left\{a_{i}=b_{i}=0\right\}$ for $i=1, \ldots, m$. It is clear that $C$ has codimension $\geq 2$ in $X$. Just as in the degree one case, since $Z$ is an affine CM normal domain, the data $\left\{\left(V, c^{n(n-1)}\right),\left(X, c^{n(n-1)}\right\}\right.$ extends to a Cartier divisor on all of $X$, so we are done by Lemma 2.2 .

As in Theorem 5.11, combining Lemma 1.16 and Lemma 5.13 implies that $W$ is reflexive and the second consequence follows.

In some sense, quantum GWAs provide an ideal setting in which to study $\mathcal{R}$-discriminants. This is because the center of a GWA is not a polynomial ring, but is reasonably nice and easy to compute. Other possible algebras to consider are quantized Weyl algebras and quantum matrix algebras.

Question 5.15. For which other families of algebras is the $\mathcal{R}$-discriminant computable? Furthermore, for which algebras is the reflexive hull of the modified discriminant ideal a principal ideal?

\section{Applications}

The purpose of this section is to show how to use $\mathcal{R}$-discriminants to study important questions in noncommutative algebra. In particular, we consider the Automorphism Problem, the Isomorphism Problem, and the Zariski Cancellation Problem for quantum GWAs. These results are known in the degree one case and so our primary interest is extending them to higher degree quantum GWAs and tensor products of quantum GWAs. To avoid some degenerate cases, for a quantum GWA $W=\mathbb{k}[t](\mathbf{x}, \mathbf{y}, \sigma, h)$ we assume for the rest of this section that

$$
h_{i} \notin \mathbb{k} \quad \text { for every } i
$$

as in many other papers. Note that (E6.0.1) implies that $W^{\times}=\mathbb{k}^{\times}$.

6.1. Automorphisms. Let $W=\mathbb{k}[t](x, y, \sigma, h)$ be a degree one quantum GWA. We recall the description of $\operatorname{Aut}(W)$ given by Suárez-Alvarez and Vivas [50]. Write $h=\sum_{i=0}^{d} c_{i} t^{i}$, where the $c_{i} \in \mathbb{k}$ and $d=\operatorname{deg}_{t}(h)$, and set $g=\operatorname{gcd}\left\{i-j \mid i<j, c_{i} c_{j} \neq 0\right\}$. Let $C_{g}$ denote the subgroup of $\mathbb{k}^{\times}$ consisting of $g$ th roots of 1 . If $h$ is a monomial, we make the convention that $g=0$ and $C_{g}=\mathbb{k}^{\times}$. For each $\gamma \in C_{g}$ and each $\mu \in \mathbb{k}^{\times}$, there is an automorphism $\eta_{\gamma, \mu}$ of $A$ given by

$$
\eta_{\gamma, \mu}(x)=\mu x, \quad \eta_{\gamma, \mu}(y)=\mu^{-1} \gamma^{d} y, \quad \eta_{\gamma, \mu}(t)=\gamma t .
$$

Let $G$ denote the subgroup of $\operatorname{Aut}(W)$ consisting of the $\eta_{\gamma, \mu}$. When $q=-1$, there is an automorphism $\Omega$ of $W$ defined by

$$
\Omega(x)=y, \quad \Omega(y)=x, \quad \Omega(t)=-t .
$$

We recover the following result of Suárez-Alvarez and Vivas using the $\mathcal{R}$-discriminant.

Proposition 6.1. [50, Theorem B] Let $q \in \mathbb{k}$ be a root of unity and $W$ be a degree one quantum $G W A$. If $q^{2} \neq 1$, then $\operatorname{Aut}(W) \cong G$. If $q=-1$, then $\operatorname{Aut}(W) \cong G \rtimes \mathbb{Z} / 2 \mathbb{Z}$ where $\mathbb{Z} / 2 \mathbb{Z}$ is generated by $\Omega$. 
Proof. It follows from (E6.0.1) that $W^{\times}=\mathbb{k}^{\times}$. Let $\phi \in \operatorname{Aut}(W)$. By Theorem 1.11(1), $\phi$ fixes the $\mathcal{R}$-discriminant up to a unit. Hence, $\phi\left(t^{n^{2}(n-1)}\right)==_{\mathbb{k}^{\times}} t^{n^{2}(n-1)}$ by Theorem 5.11, Since $W$ is a $\mathbb{Z}$-graded domain with $\operatorname{deg} x=1, \operatorname{deg} y=-1$ and $\operatorname{deg} t=0, \operatorname{deg} \phi(t)=0$. Then $\left.\phi\right|_{W_{0}}$ is an automorphism of $W_{0}=\mathbb{k}[t]$ so $\phi(t)=\gamma t$ for some $\gamma \in \mathbb{k}^{\times}$.

Observe that $\phi(x) \phi(y)=\phi(x y)=\phi(h)$ is a homogeneous element of degree 0. Hence, $\phi(x)$ and $\phi(y)$ are homogeneous elements with $\operatorname{deg} \phi(x)=-\operatorname{deg} \phi(y)$. Since $\phi(x), \phi(y), \phi(t)$ generate $W$ as an algebra, we must have $\operatorname{deg} \phi(x)= \pm 1$. So $\phi(x)=\alpha x$ or $\phi(x)=\alpha y$ for some $\alpha \in \mathbb{k}[t], \alpha \neq 0$. Similarly, $\phi(y)=\beta y$ or $\phi(y)=\beta x$ for some $\beta \in \mathbb{k}[t], \beta \neq 0$. Thus,

$$
\operatorname{deg}_{t}(x y)=\operatorname{deg}_{t}(h)=\operatorname{deg}_{t}(\phi(h))=\operatorname{deg}_{t}(\phi(x y))=\operatorname{deg}_{t}(\alpha)+\operatorname{deg}_{t}(\beta)+\operatorname{deg}_{t}(x y),
$$

so $\operatorname{deg}_{t}(\alpha)=\operatorname{deg}_{t}(\beta)=0$. Thus, $\alpha, \beta \in \mathbb{k}^{\times}$. If $\phi(x)=\alpha y$, then $0=\phi(x t-q t x)=\alpha \gamma\left(1-q^{2}\right) y t$, so $q^{2}=1$.

Case 1: Assume $\phi(x)=\alpha x$ and $\phi(y)=\beta y$ for some $\alpha, \beta \in \mathbb{k}^{\times}$. Write $h=\sum_{i=0}^{d} c_{i} t^{i}$ with $c_{i} \in \mathbb{k}$ and $c_{d} \neq 0$. Then

$$
\sum_{i=0}^{d} \alpha \beta c_{i} t^{i}=(\alpha \beta) h=(\alpha \beta) x y=\phi(x y)=\phi(h)=\sum_{i=0}^{d} c_{i} \gamma^{i} t^{i} .
$$

This implies that $\alpha \beta=\gamma^{i}$ for all $i$ such that $c_{i} \neq 0$. In particular, $\alpha \beta=\gamma^{d}$, so $\beta=\alpha^{-1} \gamma^{d}$. Now if $i<d$ and $c_{i} \neq 0$, then we have $\gamma^{d}=\gamma^{i}$, so ord $(\gamma) \mid d-i$. Hence, with $g=\operatorname{gcd}\left\{d-i \mid i<d, a_{i} \neq 0\right\}$ as above, we must have $\gamma^{g}=1$. It follows that $\phi=\eta_{\gamma, g} \in G$.

Case 2: Assume $\phi(x)=\alpha y$ and $\phi(y)=\beta x$ for some $\alpha, \beta \in \mathbb{k}^{\times}$, so that $q=-1$ by the argument after (E6.1.1). Now $\phi \circ \Omega$ is of the type in Case 1, so $\phi \circ \Omega=\eta_{-\gamma, g}$, whence $\phi=\eta_{-\gamma, g} \circ \Omega$.

Proposition 6.1 extends easily to the degree $m$ case, as long as the orders of the automorphisms $\sigma_{i}$ are pairwise coprime.

Proposition 6.2. Let $W=\mathbb{k}[t](\mathbf{x}, \mathbf{y}, \sigma, h)$ be a quantum $G W A$ of degree $m$. Suppose that each $q_{i}$ is a root of unity with $1<n_{i}=\left|\sigma_{i}\right|<\infty$ and $\operatorname{gcd}\left(n_{i}, n_{j}\right)=1$ for all $i \neq j$. Let $\phi \in \operatorname{Aut}(W)$. Then for each $i, \phi$ restricts to an automorphism of the quantum $G W A$ subalgebra $W_{i}=\mathbb{k}[t]\left(x_{i}, y_{i}, \sigma_{i}, h_{i}\right)$.

Proof. By Theorem 5.14 and similar to the proof of Proposition 6.1 $\phi(t)=\gamma t$ for some $\gamma \in \mathbb{k}$. For $i \in\{1, \ldots, n\}$, we have $\phi\left(x_{i} y_{i}\right)=\phi\left(h_{i}\right)$ and $\operatorname{deg}\left(h_{i}\right)=\mathbf{0}$. Using the $\mathbb{Z}^{n}$-grading on $W$, we have that there exists $j \in\{1, \ldots, n\}$ such that

$$
\text { (1) } \phi\left(x_{i}\right)=\alpha_{i} x_{j}, \phi\left(y_{i}\right)=\beta_{i} y_{j} \quad \text { or } \quad(2) \phi\left(x_{i}\right)=\alpha_{i} y_{j}, \phi\left(y_{i}\right)=\beta_{i} x_{j} .
$$

Suppose we are in case (1). A similar argument holds in case (2). Then

$$
0=\phi\left(x_{i} t-q_{i} t x_{i}\right)=\alpha_{i} \gamma\left(q_{j}-q_{i}\right) t x_{j} .
$$

By our hypothesis on the $q_{i}$, we have $q_{i}=q_{j}$. Hence, $j=i$. This shows that $\phi$ restricted to $W_{i}$ is of the form $\eta_{\gamma, g}$ or, when $q=-1$, possibly $\eta_{\gamma, g} \circ \Omega$.

Let $W_{1}, \ldots, W_{k}$ be a collection of degree one quantum GWAs with canonical generators $\left\{x_{i}, y_{i}, t_{i}\right\}$ and parameters $\left\{q_{i}, h_{i}\right\}$. Set $A=W_{1} \otimes \cdots \otimes W_{k}$. Note that $A$ is a degree $k$ GWA with base ring $\mathbb{k}\left[t_{1}\right] \otimes \cdots \otimes \mathbb{k}\left[t_{k}\right]$. The next proposition should be compared to [33, Theorem $\left.\mathrm{C}\right]$ in the context of quantized Weyl algebras.

Proposition 6.3. Let $A=W_{1} \otimes \cdots \otimes W_{k}$ as above. Assume each $q_{i}$ is a root of unity with $q_{i}^{2} \neq 1$. Also assume $\operatorname{deg}_{t_{i}} h_{i} \geq 2$ for all $i$. If $\phi \in \operatorname{Aut}(A)$, then the following hold: 
(1) There exists $\tau \in \mathbb{S}_{k}$ such that $\phi\left(W_{i}\right)=W_{\tau(i)}$ for all $i=1, \ldots, k$.

(2) There exists scalars $\alpha_{1}, \beta_{1}, \ldots, \alpha_{k}, \beta_{k} \in \mathbb{k}^{\times}$and a sequence $\left\{\epsilon_{1}, \ldots, \epsilon_{k}\right\} \in\{ \pm 1\}^{k}$ such that for each $i=1, \ldots, k$,

$$
\begin{aligned}
& \phi\left(x_{i}\right)=\alpha_{i} x_{\tau(i)}, \quad \phi\left(y_{i}\right)=\beta_{i} y_{\tau(i)}, \quad \text { if } \epsilon_{i}=1, \\
& \phi\left(x_{i}\right)=\alpha_{i} y_{\tau(i)}, \quad \phi\left(y_{i}\right)=\beta_{i} x_{\tau(i)}, \quad \text { if } \epsilon_{i}=-1 .
\end{aligned}
$$

Moreover, there exists scalars $\gamma_{1}, \ldots, \gamma_{k} \in \mathbb{k}^{\times}$such that

$$
h_{i}\left(\gamma_{i} t\right)= \begin{cases}\alpha_{i} \beta_{i} h_{\tau(i)}(t) & \text { if } \epsilon_{i}=1 \\ \alpha_{i} \beta_{i} h_{\tau(i)}\left(q^{-1} t\right) & \text { if } \epsilon_{i}=-1 .\end{cases}
$$

Proof. Let $\overline{\mathbb{k}}$ be the algebraic closure of $\mathbb{k}$. It is clear that we only need to show the statements for $A \otimes_{\mathbb{k}} \overline{\mathbb{k}}$. In other words, we may assume $\mathbb{k}$ is algebraically closed without loss of the generality. Let $Z$ be the center of $A$.

By Lemma 5.6, each $W_{i}$ is Auslander-Gorenstein and GK-Macaulay. Hence, $A$ is as well. Similarly, each $W_{i}$ is module-finite over its center, so $A$ is CM over $Z$. Together with Lemma 5.8 and Theorem 5.11 we may now apply Theorem 2.6 .

By induction and Theorem 2.6. the $\mathcal{R}$-discriminant of $A / Z$ is $\varrho(A / Z)=t_{1}^{N_{1}} \cdots t_{k}^{N_{k}}=: d$ for some positive integers $N_{i}$. Let $\phi \in \operatorname{Aut}(A)$. By Theorem 1.11, $\phi(d)={ }_{W} \times d$ and since $W^{\times}=\mathbb{k}^{\times}$, therefore $\phi(d)$ has degree $\mathbf{0}$ in the $\mathbb{Z}^{k}$-graded domain $A$. Since $\mathbb{Z}^{k}$ can be given a total order, this implies that each $\phi\left(t_{i}\right)$ is homogeneous of some $\mathbb{Z}^{k}$-grading. We claim that $\phi\left(t_{i}\right)=\gamma_{i} t_{\tau(i)}$ for some $\gamma_{i} \in \mathbb{k}^{\times}$ and some $\tau \in \mathbb{S}_{k}$.

After reordering, we may assume $N_{1} \geq N_{2} \geq \cdots \geq N_{k}$. Suppose $x_{i}^{p}$ is a factor of $\phi\left(t_{1}\right)$ for some $i \in\{1, \ldots, k\}$ and some $p>0$. Hence, $x_{i}^{p N_{1}}$ is a factor of $\phi(d)$. Since $\phi(d)$ has degree $\mathbf{0}$ in the $\mathbb{Z}^{k}$-grading, then it follows that $y_{i}^{p N_{1}}$ is a factor of $\phi(d)$. Then $\left(x_{i}^{p} y_{i}^{p}\right)^{N_{1}}$ is a factor of $\phi(d)$. As $A_{\mathbf{0}}=\mathbb{k}\left[t_{1}, \ldots, t_{k}\right]$ is a domain and $d$ is homogeneous in the $t_{i}$-grading, then $h_{i}=t_{i}^{q}$ for some $q \geq 2$. This implies $d$ has $t_{i}$-degree at least $p q N_{1}>N_{1}$ in $d$, a contradiction. It follows that $\phi\left(t_{1}\right)=\gamma_{1} t_{i}$ for some $i$. In this case we also obtain that $N_{i}=N_{1}$. The claim now follows by induction.

By the claim just proved above, $\phi$ preserves the polynomial subring $A_{\mathbf{0}}:=\mathbb{k}\left[t_{1}, \ldots, t_{k}\right]$. Now,

$$
h\left(\gamma_{i} t_{\tau(i)}\right)=h\left(\phi\left(t_{i}\right)\right)=\phi\left(h\left(t_{i}\right)\right)=\phi\left(x_{i} y_{i}\right)=\phi\left(x_{i}\right) \phi\left(y_{i}\right) .
$$

It follows from the $\mathbb{Z}^{k}$-grading that $\operatorname{deg}\left(\phi\left(x_{i}\right)\right)= \pm \mathbf{e}_{\tau(i)}$ for some $\tau \in \mathbb{S}_{k}$ and $\operatorname{deg}\left(\phi\left(x_{i}\right)\right)=$ $-\operatorname{deg}\left(\phi\left(y_{i}\right)\right)$. Furthermore, an argument as in (E6.1.1) show that $\phi\left(W_{i}\right)=W_{\tau(i)}$. Now it is straightforward to fill out all details.

6.2. Isomorphisms. First we reprove one case of [50, Theorem A].

Proposition 6.4. [50, Theorem $\mathrm{A}]$ Let $W=\mathbb{k}[t](x, y, \sigma, h)$ and $W^{\prime}=\mathbb{k}[T]\left(X, Y, \sigma^{\prime}, H\right)$ be degree one quantum $G W A s$ where $\sigma(t)=q t$ and $\sigma^{\prime}(T)=q^{\prime} T$. Assume $q, q^{\prime} \neq 1$ are roots of unity. If $\Phi: W \rightarrow W^{\prime}$ is an isomorphism, then $q^{\prime}=q^{ \pm 1}$ and there exists $\gamma, \mu \in \mathbb{k}^{\times}$such that

$$
h(\gamma t)= \begin{cases}\mu H(T) & \text { if } q^{\prime}=q \\ \mu H\left(q^{-1} T\right) & \text { if } q^{\prime}=q^{-1} .\end{cases}
$$

Moreover, if $q^{\prime}=q^{ \pm 1}$ and there exist $\gamma, \mu$ satisfying the above condition, then $W \cong W^{\prime}$.

Proof. It is easy to verify that an isomorphism $W \rightarrow W^{\prime}$ exists under these conditions. So assume $\Phi: W \rightarrow W^{\prime}$ is an isomorphism. An argument as in Proposition 6.1 shows that $\Phi(t)=\gamma T$ for some 
$\gamma \in \mathbb{k}^{\times}$, and that either $\Phi(x)=\alpha X$ and $\Phi(y)=\beta Y$, or else $\Phi(x)=\alpha Y$ and $\Phi(y)=\beta X$ for some $\alpha, \beta \in \mathbb{k}^{\times}$. In the first case, we have

$$
0=\Phi(x t-q t x)=\alpha \gamma(X T-q T X)=\alpha \gamma\left(q^{\prime}-q\right) T X,
$$

so $q^{\prime}=q$, and

$$
h(\gamma t)=\Phi(h(t))=\Phi(x y)=\Phi(x) \Phi(y)=(\alpha \beta) X Y=(\alpha \beta) h(T) .
$$

The second case is similar.

Now we extend this to higher degree quantum GWAs.

Proposition 6.5. Let $W=\mathbb{k}[t](\sigma, h)$ and $W^{\prime}=\mathbb{k}[T]\left(\sigma^{\prime}, H\right)$ be quantum $G W A$ s of degree $m$ and $m^{\prime}$, respectively, with parameters $\left(q_{1}, \ldots, q_{m}\right)$ and $\left(q_{1}^{\prime}, \ldots, q_{m^{\prime}}^{\prime}\right)$, respectively, such that $q_{i}^{2},\left(q_{i}^{\prime}\right)^{2} \neq 1$ for all $i$. Suppose both $W$ and $W^{\prime}$ satisfy the hypothesis of Proposition 6.2. If $\phi: W \rightarrow W^{\prime}$ is an isomorphism, then $m=m^{\prime}$ and there exists $\tau \in \mathbb{S}_{m}$ such that $W_{i} \cong W_{\tau(i)}^{\prime}$.

Proof. By Lemma 5.4, $m+1=$ GKdim $W=$ GKdim $W^{\prime}=m^{\prime}+1$, so $m=m^{\prime}$. Denote the canonical generators of $W$ by $x_{1}, y_{1}, \ldots, x_{m}, y_{m}$ and those of $W^{\prime}$ by $X_{1}, Y_{1}, \ldots, X_{m}, Y_{m}$. Let $\phi: W \rightarrow W^{\prime}$ be the given isomorphism, then using similar arguments to the above we have $\phi(t)=\gamma T$ for some $\gamma \in \mathbb{k}^{\times}$. Then $\phi\left(x_{i}\right) \phi\left(y_{i}\right)$ has degree $\mathbf{0}$ for each $i$. Hence, there is some index $j$ such that $\phi\left(x_{i}\right)=\alpha_{i} X_{j}$ and $\phi\left(y_{i}\right)=\beta_{i} Y_{j}$, or else $\phi\left(x_{i}\right)=\alpha_{i} Y_{j}$ and $\phi\left(y_{i}\right)=\beta_{i} X_{j}$. We now refer to Proposition 6.4 for a description of the isomorphism $W_{i} \rightarrow W_{j}$.

6.3. Cancellation. The Zariski cancellation problem (ZCP) asks whether an algebra isomorphism $A[x] \cong B[x]$ implies the existence of an algebra isomorphism $A \cong B$; if so, then $A$ is called cancellative [Definition 0.7. Solving the ZCP for various classes of noncommutative algebras has attracted much recent interest [5, 6, 21, 34, 35]. In this subsection we use $\mathcal{R}$-discriminants to prove that degree $m$ quantum GWAs, as well as their tensor products, are cancellative. For simplicity, we assume that char $\mathbb{k}=0$ in this subsection.

Makar-Limanov showed that the cancellation property is inherently tied to the study of locally nilpotent derivations [36]. We we denote the set of locally nilpotent derivations of an algebra $A$ by $\operatorname{LND}(A)$. We say $A$ is $L N D$-rigid if $\operatorname{LND}(A)=\{0\}$ [6, p.1711].

A degree one quantum GWA $W$ is cancellative by [6, Corollary 3.7 (2)]. Alternatively, one recovers this result by combining [6, Theorem 3.6] and [50, Lemma 2.1].

Theorem 6.6. Let $W=\mathbb{k}[t](\mathbf{x}, \mathbf{y}, \sigma, h)$ be a quantum $G W A$ of degree $m$ and $Z$ its center.

(1) Suppose that each $q_{i}$ is a root of unity with $n_{i}=\left|\sigma_{i}\right|<\infty, \operatorname{gcd}\left(n_{i}, n_{j}\right)=1$ for all $i \neq j$, and $n_{i}>1$ for at least one $i$. Then $W$ is cancellative.

(2) Let $A$ be the tensor product of finitely many algebras in part (1). Then $A$ is cancellative.

Proof. (1) By [6, Theorem 3.6], it suffices to prove that $W$ is LND-rigid. Let $\delta \in \operatorname{LND}(W)$ and let $d$ denote the $\mathcal{R}$-discriminant of $W$ given in Theorem 5.14. Since we assume (E6.0.1), $W^{\times}=\mathbb{k}^{\times}$. By Theorem $1.11(2), \delta(d)=0$. By the Leibniz rule and because $W$ is a domain, $\delta(t)=0$. Since $x_{i} y_{i} \in \mathbb{k}[t]$, then by [17, Lemma 7.4] and [36, p. 4], $\delta\left(x_{i}\right)=\delta\left(y_{i}\right)=0$. Thus, $\operatorname{LND}(A)=\{0\}$.

(2) The result for tensor products of quantum GWAs is similar. First we need to pass to the case when $\mathbb{k}$ is algebraically closed. Then $d:=\varrho(A / Z(A))$ exists by Theorem $2.6(3)$ and 5.14 , Then we can copy the proof of part (1) with minor changes. 


\section{REFERENCES}

[1] E. Akalan. On rings whose reflexive ideals are principal. Comm. Algebra, 38(9):3174-3180, 2010.

[2] V. V. Bavula. Generalized Weyl algebras and their representations. Algebra i Analiz, 4(1):75-97, 1992.

[3] V. V. Bavula. Global dimension of generalized Weyl algebras. In Representation theory of algebras (Cocoyoc, 1994), volume 18 of CMS Conf. Proc., pages 81-107. Amer. Math. Soc., Providence, RI, 1996.

[4] V. V. Bavula and D. A. Jordan. Isomorphism problems and groups of automorphisms for generalized Weyl algebras. Trans. Amer. Math. Soc., 353(2):769-794, 2001.

[5] J. Bell, M. Hamidizadeh, H. Huang, and H. Venegas. Noncommutative analogues of a cancellation theorem of Abhyankar, Eakin, and Heinzer. Beitr. Algebra Geom., 62(2):295-315, 2021.

[6] J. Bell and J. J. Zhang. Zariski cancellation problem for noncommutative algebras. Selecta Math. (N.S.), 23(3):1709-1737, 2017.

[7] G. Benkart and M. Ondrus. Whittaker modules for generalized Weyl algebras. Represent. Theory, 13:141-164, 2009.

[8] S. Bouchiba and S. Kabbaj. Tensor products of Cohen-Macaulay rings: solution to a problem of Grothendieck. J. Algebra, 252(1):65-73, 2002.

[9] K. A. Brown and K. R. Goodearl. Homological aspects of Noetherian PI Hopf algebras of irreducible modules and maximal dimension. J. Algebra, 198(1):240-265, 1997.

[10] K. A. Brown and C. R. Hajarnavis. Homologically homogeneous rings. Trans. Amer. Math. Soc., 281(1):197-208, 1984.

[11] K. A. Brown, C. R. Hajarnavis, and A. B. MacEacharn. Rings of finite global dimension integral over their centres. Comm. Algebra, 11(1):67-93, 1983.

[12] K. A. Brown and M. J. Macleod. The Cohen Macaulay property for noncommutative rings. Algebr. Represent. Theory, 20(6):1433-1465, 2017.

[13] K. A. Brown and M. T. Yakimov. Azumaya loci and discriminant ideals of PI algebras. Adv. Math., 340:1219$1255,2018$.

[14] S. Çeken, J. H. Palmieri, Y.-H. Wang, and J. J. Zhang. The discriminant controls automorphism groups of noncommutative algebras. Adv. Math., 269:551-584, 2015.

[15] S. Çeken, J. H. Palmieri, Y.-H. Wang, and J. J. Zhang. The discriminant criterion and automorphism groups of quantized algebras. Adv. Math., 286:754-801, 2016.

[16] S. Çeken, J. H. Palmieri, Y.-H. Wang, and J. J. Zhang. Invariant theory for quantum Weyl algebras under finite group action. In Lie Algebras, Lie Superalgebras, Vertex Algebras and Related Topics, volume 92 of Proc. Sympos. Pure Math., pages 119-134. Amer. Math. Soc., Providence, RI, 2016.

[17] K. Chan, A. A. Young, and J. J. Zhang. Discriminant formulas and applications. Algebra Number Theory, 10(3):557-596, 2016.

[18] K. Chan, A. A. Young, and J. J. Zhang. Discriminants and automorphism groups of Veronese subrings of skew polynomial rings. Math. Z., 288(3-4):1395-1420, 2018.

[19] E. Ebrahim. The prime spectrum and representation theory of the $2 \times 2$ reflection equation algebra. Comm. Algebra, 47(3):1153-1196, 2019.

[20] J. Gaddis, E. Kirkman, and W. F. Moore. On the discriminant of twisted tensor products. J. Algebra, 477:29-55, 2017.

[21] J. Gaddis and X. Wang. The Zariski cancellation problem for Poisson algebras. J. Lond. Math. Soc. (2), 101(3):1250-1279, 2019.

[22] J. Gaddis, R. Won, and D. Yee. Discriminants of Taft Algebra Smash Products and Applications. Algebr. Represent. Theory, 22(4):785-799, 2019.

[23] V. Ginzburg. Calabi-Yau algebras. arXiv preprint math/0612139, 2006.

[24] R. Hartshorne. Generalized divisors on Gorenstein schemes. In Proceedings of Conference on Algebraic Geometry and Ring Theory in honor of Michael Artin, Part III (Antwerp, 1992), volume 8 (3), pages 287-339, 1994.

[25] T. J. Hodges. Morita equivalence of primitive factors of $U(\mathrm{sl}(2))$. In Kazhdan-Lusztig theory and related topics (Chicago, IL, 1989), volume 139 of Contemp. Math., pages 175-179. Amer. Math. Soc., Providence, RI, 1992.

[26] T. J. Hodges. Noncommutative deformations of type-A Kleinian singularities. J. Algebra, 161(2):271-290, 1993.

[27] D. A. Jordan. Krull and global dimension of certain iterated skew polynomial rings. In Abelian groups and noncommutative rings, volume 130 of Contemp. Math., pages 201-213. Amer. Math. Soc., Providence, RI, 1992. 
[28] A. Joseph. A generalization of Quillen's lemma and its application to the Weyl algebras. Israel J. Math., 28(3):177-192, 1977.

[29] E. Kirkman and J. Kuzmanovich. Fixed subrings of Noetherian graded regular rings. J. Algebra, 288(2):463-484, 2005.

[30] G. R. Krause and T. H. Lenagan. Growth of algebras and Gelfand-Kirillov dimension, volume 22 of Graduate Studies in Mathematics. American Mathematical Society, Providence, RI, revised edition, 2000.

[31] R. S. Kulkarni. Down-up algebras and their representations. J. Algebra, 245(2):431-462, 2001.

[32] T. Levasseur and J. T. Stafford. The quantum coordinate ring of the special linear group. J. Pure Appl. Algebra, 86(2):181-186, 1993.

[33] J. Levitt and M. Yakimov. Quantized Weyl algebras at roots of unity. Israel J. Math., 225(2):681-719, 2018.

[34] O. Lezama, Y.-H. Wang, and J. J. Zhang. Zariski cancellation problem for non-domain noncommutative algebras. Math. Z., 292(3-4):1269-1290, 2019.

[35] D.-M. Lu, Q.-S. Wu, and J. J. Zhang. A Morita cancellation problem. Canad. J. Math., 72(3):708-731, 2020.

[36] L. Makar-Limanov. Locally nilpotent derivations, a new ring invariant and applications. Preprint, pages 1-60, 1998.

[37] J. C. McConnell and J. C. Robson. Noncommutative Noetherian rings, volume 30 of Graduate Studies in Mathematics. American Mathematical Society, Providence, RI, revised edition, 2001. With the cooperation of L. W. Small.

[38] B. Nguyen, K. Trampel, and M. Yakimov. Noncommutative discriminants via Poisson primes. Adv. Math., 322:269-307, 2017.

[39] B. Nguyen, K. Trampel, and M. Yakimov. Root of unity quantum cluster algebras and discriminants. arXiv:2012.02314, 2021.

[40] I. Reiner. Maximal orders, volume 28 of London Mathematical Society Monographs. New Series. The Clarendon Press, Oxford University Press, Oxford, 2003. Corrected reprint of the 1975 original, With a foreword by M. J. Taylor.

[41] L. Richard and A. Solotar. Isomorphisms between quantum generalized Weyl algebras. J. Algebra Appl., 5(3):271285, 2006.

[42] A. L. Rosenberg. Noncommutative algebraic geometry and representations of quantized algebras, volume 330 of Mathematics and its Applications. Kluwer Academic Publishers Group, Dordrecht, 1995.

[43] L. Rowen and D. J. Saltman. Tensor products of division algebras and fields. J. Algebra, 394:296-309, 2013.

[44] L. Silver. Tame orders, tame ramification and Galois cohomology. Illinois J. Math., 12:7-34, 1968.

[45] S. P. Smith. A class of algebras similar to the enveloping algebra of $\mathfrak{s l}(2)$. Transactions of the American Mathematical Society, 322(1):285-314, 1990.

[46] The Stacks Project Authors. Stacks Project. https://stacks.math.columbia.edu, 2018.

[47] J. T. Stafford. Homological properties of the enveloping algebra U( $\left.\mathrm{Sl}_{2}\right)$. Math. Proc. Cambridge Philos. Soc., 91(1):29-37, 1982.

[48] J. T. Stafford and M. Van den Bergh. Noncommutative resolutions and rational singularities. Michigan Math. $J ., 57: 659-674,2008$. Special volume in honor of Melvin Hochster.

[49] J. T. Stafford and J. J. Zhang. Homological properties of (graded) Noetherian PI rings. J. Algebra, 168(3):9881026, 1994.

[50] M. Suárez-Alvarez and Q. Vivas. Automorphisms and isomorphisms of quantum generalized Weyl algebras. J. Algebra, 424:540-552, 2015.

[51] M. Van den Bergh. Non-commutative crepant resolutions. In The legacy of Niels Henrik Abel, pages 749-770. Springer, Berlin, 2004.

[52] C. Walton, X. Wang, and M. Yakimov. Poisson geometry of PI three-dimensional Sklyanin algebras. Proc. Lond. Math. Soc. (3), 118(6):1471-1500, 2019.

[53] C. Walton, X. Wang, and M. Yakimov. Poisson geometry and representations of PI 4-dimensional Sklyanin algebras. Selecta Math. (N.S.), 27(5):Paper No. 99, 60, 2021.

[54] X. Zhao, Q. Mo, and Y. Zhang. Gelfand-Kirillov dimension of generalized Weyl algebras (in memory of Guenter Rudolf Krause (1941-2015)). Comm. Algebra, 46(10):4403-4413, 2018. 
(Chan) Department of Mathematics, Box 354350, University of Washington, Seattle, Washington 98195, USA

Email address: kenhchan@math.washington.edu, ken.h.chan@gmail.com

(Gaddis) Department of Mathematics, Miami University, Oxford, Ohio 45056, USA

Email address: gaddisj@miamioh.edu

(Won) Department of Mathematics, The George Washington University, Washington, DC 20052, USA

Email address: robertwon@gwu.edu

(Zhang) Department of Mathematics, Box 354350, University of Washington, Seattle, Washington 98195, USA

Email address: zhang@math.washington.edu 